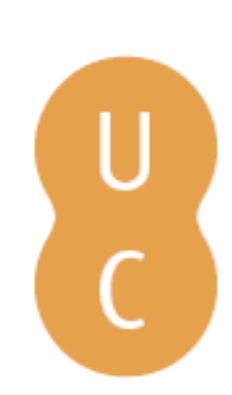

\title{
nommalina
}

\section{La virtù al femminile}

Autor(es): Melandri, Eleonora

Publicado por: Imprensa da Universidade de Coimbra; Katholieke Universiteit Leuven

URL

persistente:

URI:http://hdl.handle.net/10316.2/32106

DOI:

DOI:http://dx.doi.org/10.14195/978-989-26-0462-6_13

Accessed : $\quad$ 26-Apr-2023 16:07:47

A navegação consulta e descarregamento dos títulos inseridos nas Bibliotecas Digitais UC Digitalis, UC Pombalina e UC Impactum, pressupõem a aceitação plena e sem reservas dos Termos e Condições de Uso destas Bibliotecas Digitais, disponíveis em https://digitalis.uc.pt/pt-pt/termos.

Conforme exposto nos referidos Termos e Condições de Uso, o descarregamento de títulos de acesso restrito requer uma licença válida de autorização devendo o utilizador aceder ao(s) documento(s) a partir de um endereço de IP da instituição detentora da supramencionada licença.

Ao utilizador é apenas permitido o descarregamento para uso pessoal, pelo que o emprego do(s) título(s) descarregado(s) para outro fim, designadamente comercial, carece de autorização do respetivo autor ou editor da obra.

Na medida em que todas as obras da UC Digitalis se encontram protegidas pelo Código do Direito de Autor e Direitos Conexos e demais legislação aplicável, toda a cópia, parcial ou total, deste documento, nos casos em que é legalmente admitida, deverá conter ou fazer-se acompanhar por este aviso.

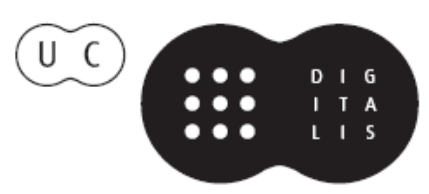




\title{
Puilosophy in Society \\ Virtues and Values in Plutarch
}

\author{
José Ribeiro Ferreira \\ LUC VAN DER STOCKT \\ Maria do Céu Fialho
}

Editors

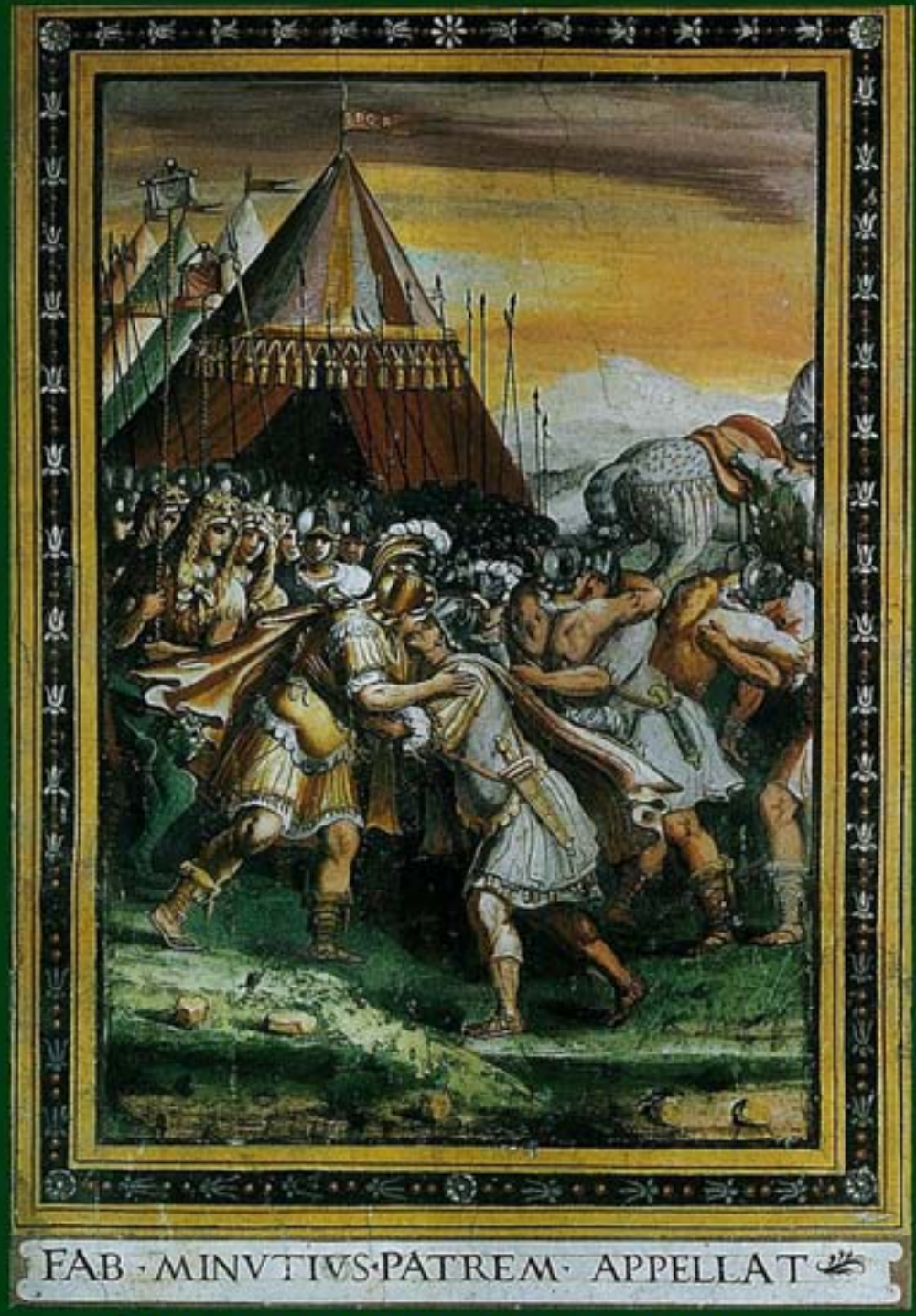

KATHOLIEKE UNIVERSITEIT LEUVEN IMPRENSA DA UNIVERSIDADE DE COIMBRA Leuven-Coimbra, 2008 
(Página deixada propositadamente em branco) 
José Ribeiro Ferreira, Luc van der Stockt \& Maria do Céu Fialho

EDITORS

\section{PhILOSOPHY IN Society}

\section{Virtues and Values in Plutarch}

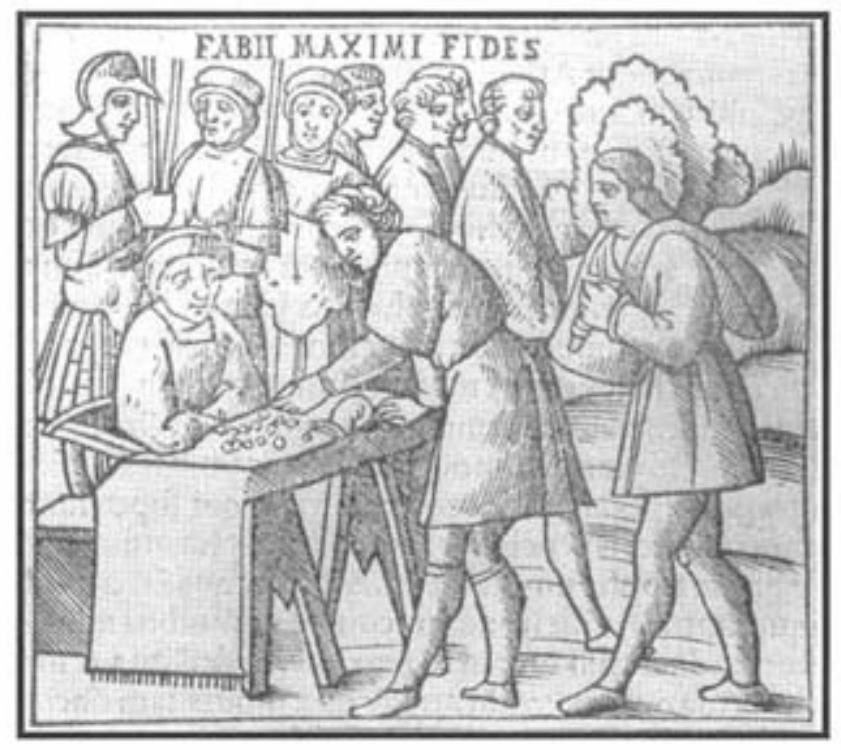

Fabius Maximus' Loyalty

Vitae Plutarchi Cheronei novissime post Jodocum Badium Ascensium longe diligentius repositae maioreque diligentia castigatae, cum copiosiore verioreque indice, nec non cum Aemilii Probi vitis, una cum figuris, suis locis apte dispositis, Venetiis 1516 , fol . 65v

\section{LEUVEN - CoIMBRA \\ 2008}

KATHOLIEKE UNIVERSITEIT LEUVEN

IMPRENSA DA UNIVERSIDADE DE COIMBRA 


\title{
First published 2008
}

\section{(C) Universiteit KATHOlieke Leuven \\ (c) Universidade Coimbra}

\author{
Published by \\ IMPRENSA DA UNIVERSIDADE COIMBRA \\ Imprensa da Universidade de Coimbra \\ Rua da Ilha, $\mathrm{n}^{\circ} 1$ \\ 3000-033 Coimbra (Portugal) \\ Email: imprensauc@ci.uc.pt \\ URL: http://www.uc.pt/imprensa_uc
}

ISBN: 972-989-8074-73-7

Legal Deposit: MA-140-2009

\author{
Printed in Spain by \\ IMAGRAF IMPRESORES, S.A. \\ c/ Nabucco 14 \\ 29006 Málaga \\ Tfno. 952328597
}

\section{Frontispiece:}

Fabius Maximus and Minucius (Francesco da Siena, Grottaferrata, Palazzo Abbaziale). We are grateful to the Archimandrita of the "Monastero Esarchico di Santa Maria di Grottaferrata", P. Emiliano Fabbricatore, for the authorization to reproduce this picture. 


\title{
La virtù al femminile
}

\author{
Eleonora Melandri \\ UNIVERSITÀ di FirENZE
}

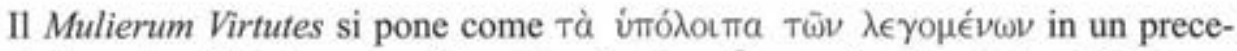
dente incontro di Plutarco con Clea $\epsilon$ is Tò $\mu$ ía

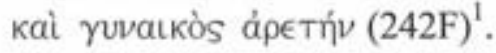

L' unicità di virtù maschile e femminile non è una novità in assoluto perchè è possibile tracciare una storia di questo motivo all' interno della filosofia greca da Antistene a Diogene di Sinope, a Epicuro e soprattutto all' antico stoicismo senza

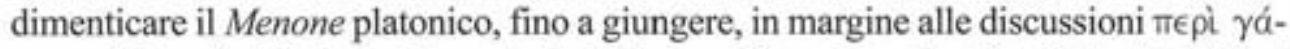
$\mu o v$, a Musonio Rufo con la sua tesi dell' uguaglianza morale e intellettuale dei due

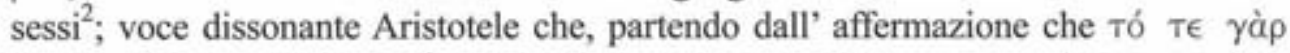

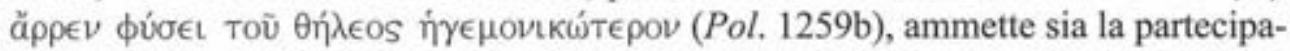

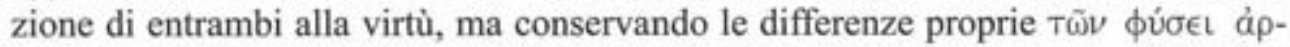
Xoú́v $\omega \nu$, sia la partecipazione alle virtù etiche, ma non allo stesso modo, ma per quanto serve a ciascuno per assolvere la sua funzione, cosicchè si conclude che, se la virtù

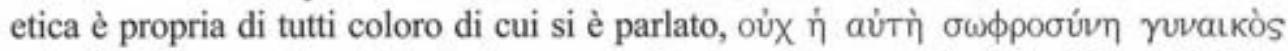

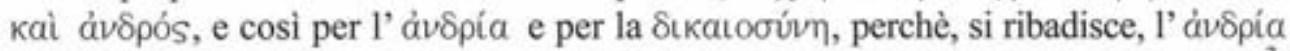

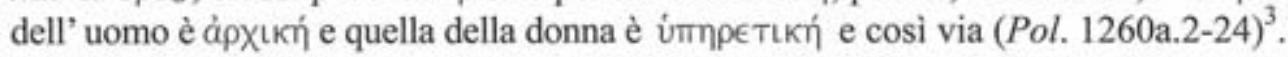

Ritornando al passo di Plutarco, possiamo leggere, dopo l' affermazione citata,

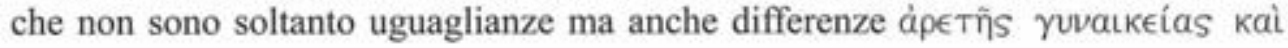

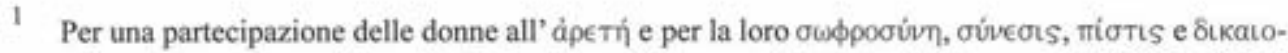

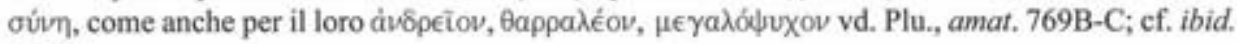
$767 \mathrm{~B}$.

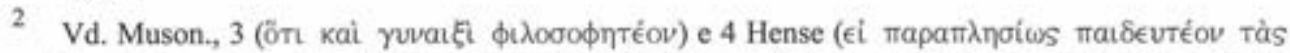
Avyatépas toís viois). Giustamente PH. A. Stadter, 1965, p. 5 sottolinea che per Musonio una donna studia filosofia per meglio assolvere i suoi compiti femminili.

3

Cf. Arist., Pol. 1277b.17-25.

José Ribeiro Ferreira, Luc VAn der Stockt \& Maria do Céu Fialho (Edd.), Philosophy in Society - Virtues and Values in Plutarch, Leuven-Coimbra, 2008, pp. 173-194. 


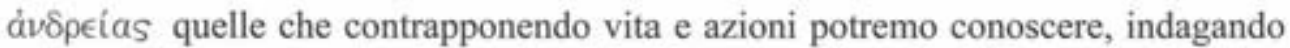

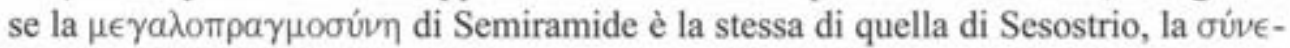

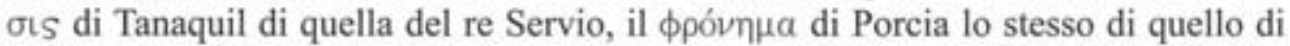

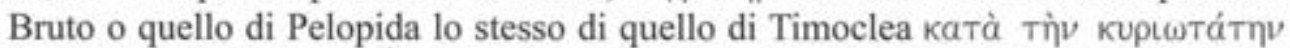

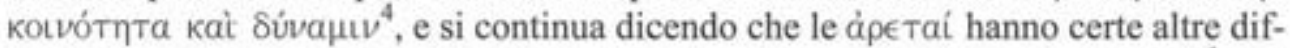

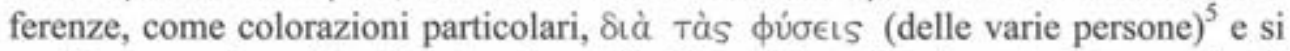
conformano ai sussistenti costumi, costituzione dei corpi, alimentazione, modo di vita (243B-D). Il principio è ben conosciuto dalle Vite Parallele: le medesime virtù manifestano se stesse in differenti individui differentemente. Ma guardiamo come Plutarco organizza in maniera sottile il suo successivo discorso: sono posti a confronto uomini con uomini per l'ả donne per la $\phi \iota \lambda a \nu \delta \rho i a$ e la $\mu \in \gamma a \lambda \circ \phi \rho \circ \sigma u ́ v \eta$ : non viene affermata una differenza di genere, ma un' ambiguità di fondo rimane, visto che il confronto interessa virtù che, fatta eccezione per la $\mu € \gamma a \lambda \circ \phi \rho \circ \sigma u ́ v \eta$, sono tradizionalmente caratteristiche dei due diversi generi ${ }^{6}$. Plutarco conclude infine dicendo che questo non vuol dire creare

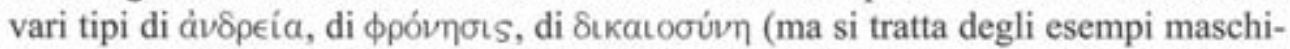

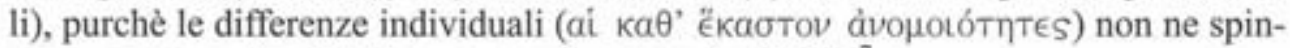
gano qualcuno fuori dalla sua propria categoria (243D) ${ }^{7}$.

Sulla scorta di questa ambiguita ${ }^{8}$, passo a render conto delle attestazioni relative

4

5

J. BOULOGNE, 2002, p. 41 traduce "en prenant la notion de mérite dans son sens le plus général"; PH. A. StADTER, 1965, p. 10 "according to most important common element and quality"; F. C. BABBrr, 1931, p. 479 "with due regard to the most important points of identity and influence". Io preferirei tradurre "secondo la fondamentale comune potenza",

PH. A. Stadter, 1965, p. 10 traduce "constitutions"; PH. A. Stadter, 1999, p. 177 "nature"; J. BOULOGNE, 2002, p. 41 "caractère".

PH.A. Stadter, 1965, p. 10 nota i due diversi tipi di confronto, ma egli commenta che per Plutarco i due tipi di confronto sono essenzialmente gli stessi perchè la virtù delle donne è uguale a quella dell' uomo; egli ipotizza inoltre che il confronto sia in collegamento con la struttura del Mulierum virtutes: un catalogo di virtuose azioni femminili senza nessun parallelo con quelle maschili.

PH. A. StadtER, 1965, p. 10 traduce "do not force one out of its proper category"; J. BOULOGNE, 2002, p. 42 "ne forcent à en exclure aucune de la définition propre"; F. C. BABBrr, 193I, p. 479 "if only the individual dissimilarities exclude no one of these from receiving its appropriate rating".

Possiamo notare che le azioni femminili presentate in Mulierum virtutes nascono o per adesione a ordini maschili, o in sostituzione di uomini assenti, vili, incapaci, immorali, e molto spesso come reazione alla propria castità violata, all' uccisione del marito (PH. A. STADTER, 1999, p. 178 n. 19 rileva che soltanto due storie, la n. 11 e la n. 12, nascono direttamente dalle donne); vengono prese le armi solo nelle storie n. 3 e n. 4 , e si fa ricorso piuttosto alle armi della persuasione e dell' inganno, all' arte della seduzione o si fa perno sull' attrazione suscitata. Importante la sottolineatura che fa PH. A Stadter, 1999, p. 179 sul fatto che le donne in questi esempi agiscono come salvatrici in tempi di crisi, ma fino a che gli uomini possono riassumere il loro ruolo dominante; ef. $\mathrm{L}$. FOXHAL.L, 1999, p. 150. 
alla $\sigma \omega \phi \rho \circ \sigma u ́ v \eta$ in Plutarco sulla base delle ricorrenze di $\sigma \omega ́ \phi \rho \omega \nu, \sigma \omega \phi \rho o ́ v \omega s$,

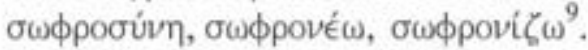

Lo stesso Plutarco offre nelle sue opere una definizione di $\sigma \omega \phi \rho \circ \sigma u ́ v \eta^{10}$ che nel

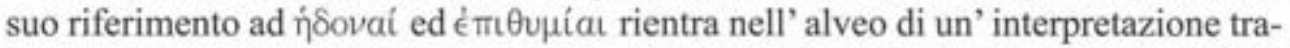
dizionale e popolare (che già lo stesso Aristotele aveva recuperato in opposizione all' interpretazione platonica), ma che rivela la sua matrice peripatetica ${ }^{11}$ nel momento in cui Plutarco la presenta come medietà di passioni e insiste sulla diffe-

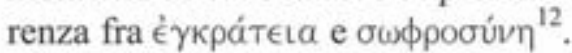

Il collegamento critico di Plutarco con Platone ha portato spesso traduttori e

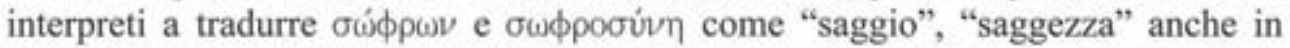
applicazione a personaggi femminili; ma dalla mia analisi generale, nelle cinquan-

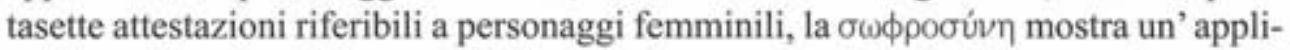
cazione alla sfera della sessualità o più largamente della fedeltà sessuale, con l' esclusione di soli sette casi.

Offro un elenco e una sintetica presentazione delle ricorrenze analizzate.

In relazione a $\sigma \omega \phi \rho \circ \sigma u ́ v \eta$ :

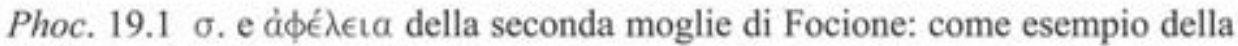
sua á $\phi \dot{\lambda} \lambda \in\llcorner a$ il fatto che si facesse accompagnare da una sola serva e che riconoscesse come unico ornamento Focione; Ant. 87.6 di una figlia di Antonio lodata per $\sigma$. e bellezza (vd. p. 183); (apophth. Lac.) 228B tanto grande a quei tempi la $\sigma$. delle donne laconiche che una loro infedeltà inconcepibile; ibid. 242B la patria $\sigma$. è la

Espressioni del tipo éàv $\sigma \omega \phi \rho \omega \nu \bar{̃}$ mantengono il significato ormai acquisito di "se hai senno", mentre $\sigma \omega \phi \rho v^{\prime}(\zeta)$ continua ad avere in molti casi il significato correttivo che era proprio dell' oratoria.

10

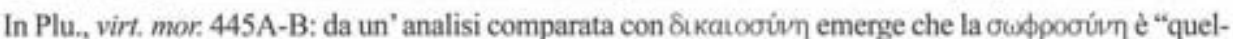

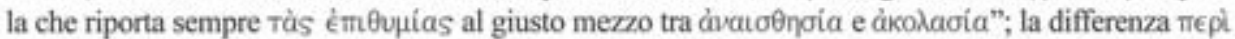

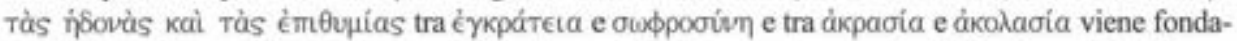
ta sul fatto che non è la stessa parte dell' anima quella che per natura svolge la funzione di desiderare e quella di giudicare e cosi si ha la owфpocivin quando la ragione "guida e govema la passione come un animale mite e docile alle redini, arrendevole nei desideri e pronto ad accogliere di buon grado la misura giusta e conveniente" (trad. F. BECCHI). In Plu., bruta anim. 989B Grillo definisce la $\sigma$. una

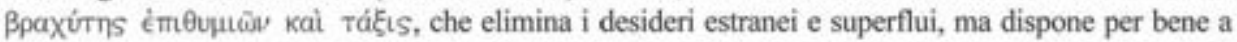
tempo opportuno e con moderazione quelli necessari. In Plu., fort. 97E il discorso verte fondamental-

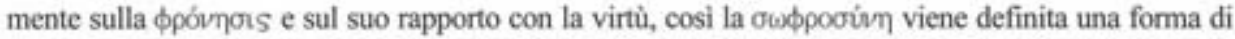

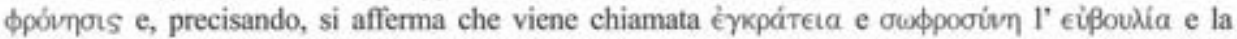

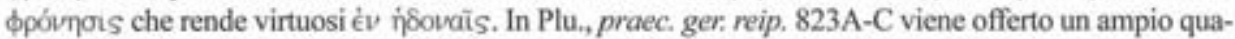
dro comportamentale del бúxpluv istituzionale; vd. pp. 185-186.

11 Cf. anche Plu., virt. mor. 443C. Vd. F, BECCH, 1990, pp. 169-170 n. 18, con il rimando a MM1186a. 


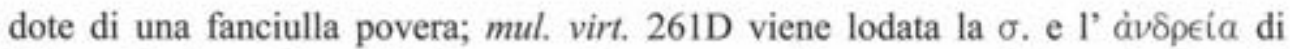
Erisso, che ha ucciso colui che, preso da amore per lei, ne aveva fatto perire il marito (vd. mul. virt. 260F); coh. ira 462A quando c' è l' ira i mariti non riescono a sop-

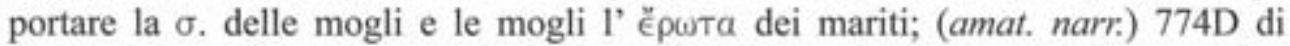
Calliroe che si distingue per bellezza e $\sigma$. (vd. p. 183); bruta anim. 989A riferimento alla $\sigma$. di Penelope all' interno di un discorso sulla continenza sessuale.

Utilizzazione dell' aggettivo $\sigma \omega ́ \phi \rho \omega \nu$, dell' avverbio $\sigma \omega \phi \rho o ́ \nu \omega s$ e del verbo $\sigma \omega \phi \rho \circ \epsilon_{\bar{L}} \nu$ :

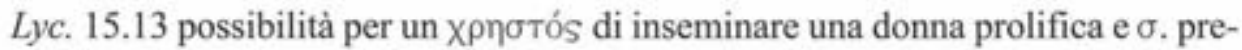
via richiesta al marito; Sol. 20.5 il rapporto tre volte al mese con la moglie é $\pi i ́ k \lambda \epsilon-$ pos segno di riguardo verso una moglie $\sigma$.; Aem. 5.2 la moglie è $\sigma$., bella e prolifica e il divorzio è immotivato (cf. coniug. praec. 141A); Sull. 35.9 Silla non sposò

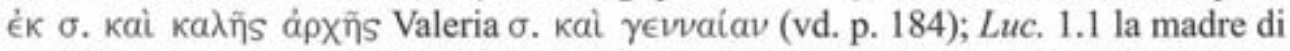

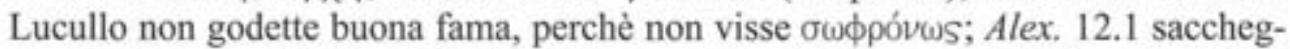

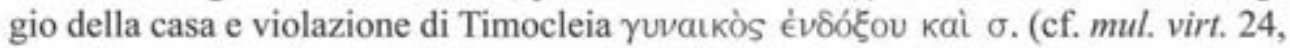
dove è esposta la vicenda di Timossena); ibid. 21.5 rispetto di Alessandro per donne $\gamma \in v$ vaiaıs kai $\sigma$. che egli difende da atti vergognosi (vd. p. 184); Caes. 9.3 la sor-

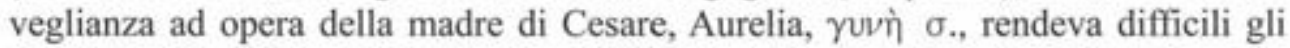
incontri tra Pompeia e Clodio; Cleom. 43.2 Cleomene addolorato per la morte $\kappa a \lambda$ -

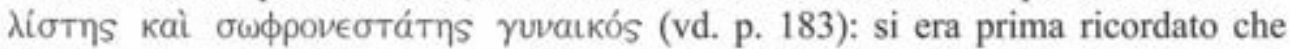
Cleomene anche durante le campagne militari tornava a Sparta perchè innamorato della moglie; Demetr: 25.9 Demetrio si difende dalle offese dicendo che la sua

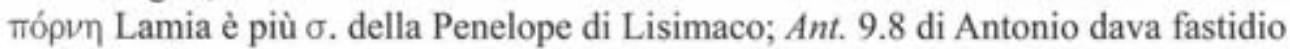

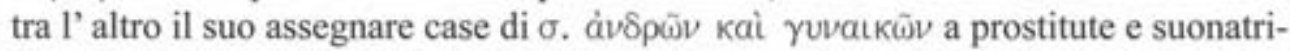
ci; aud. poet. 32C le parole usate da Hom. Od. 3.265-266 per spiegare l' iniziale

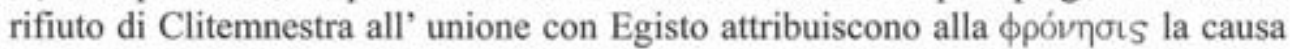
del $\sigma \omega \phi \rho о \nu \in \check{\nu}$; cap. ex inim. ut. 90B la moglie $\sigma$. кà ăkakos non ha mai rimproverato al marito il suo alito cattivo perchè pensava che tutti gli uomini l' avessero cosi (limitatezza della sua esperienza con l' altro sesso); coniug. praec. 139C la moglie $\sigma$. deve farsi vedere solo in compagnia del marito; ibid. $139 \mathrm{C}$ la donna $\sigma$. quando si spoglia si veste del suo pudore; $i b i d .140 \mathrm{C}$ il marito modella la moglie: I' amante dei piaceri la rende etera e dissoluta, l' amante del bello e buono $\sigma$. е коб-

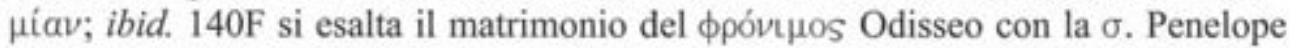
(cf. brut. anim. 988F e 989A); ibid. 141A un famoso Romano viene criticato per

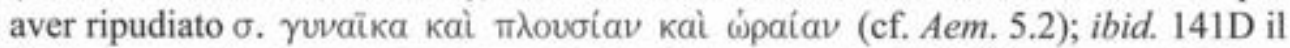
vizio deturpa il viso e la brutta guardandosi allo specchio dirà: "Che cosa sarà di me se non saprò essere $\sigma . ?$ " e la bella: "Che cosa sarà di me se saprò essere anche $\sigma . ?$; ibid. 141F-142A la moglie $\sigma$. deve usare la grazia nei rapporti col marito, senza adirarsi perchè ella $\sigma \omega \phi \rho o v \in \tilde{i}$ (cf. amat. 753C); ibid. 142C il marito di fronte ad una moglie $\sigma$. e severa deve considerare che la persona con cui si unisce non può essere al contempo moglie legittima ed etera; $i b i d .142 \mathrm{C}$ la $\sigma$. in pubblico non solo non 
deve mostrare il braccio, ma nemmeno fare sentire la sua voce, che rivela tutto di lei; ibid. $143 \mathrm{~F}$ la moglie non deve ascoltare le malelingue, secondo cui il marito

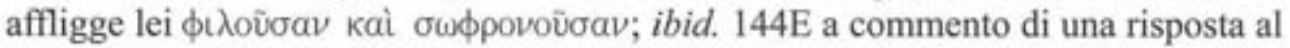
libertino re Filippo, si afferma che la moglie legittima al buio, diversamente da una

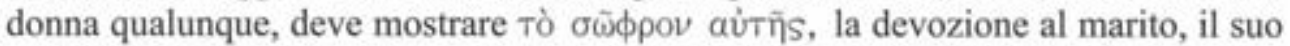
ritegno e il suo affetto; $\mathrm{mul}$. virt. 257E si parla di Camma ammirata per la sua bellezza, ma soprattutto per la sua virtù e non soltanto $\sigma$. e $\phi i \lambda a v \delta \rho o s$ etc. (cf. amat. 768B-D sulla nobiltà, bellezza, onestà di Camma che, dopo l' uccisione dell' amato marito, non si lasciava avvicinare da nessuno e rifiutò i molti pretendenti); ibid. $260 \mathrm{~F}$ dopo la morte di Arcesilao, piuttosto che al figlio ancora giovane, molti erano

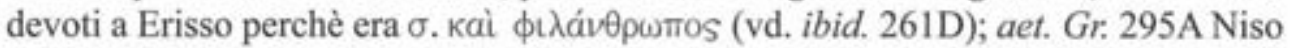

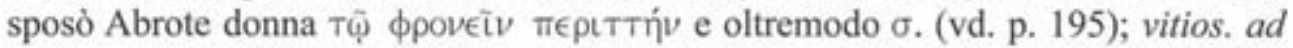
inf. suff. $499 \mathrm{C}$ le vedove indiane $\phi i \lambda a v \delta \rho \rho \mathrm{L}$ kai $\sigma$. fanno a gara per salire sulla pira del marito (vd. p. 184); gen. Socr. 598C le donne accompagnavano padri e mariti senza che nessuno le fermasse, per l' impressione suscitata dalla pietà, dalle lacrime e dalle suppliche di donne $\sigma$.; amat. $752 \mathrm{C}$ per Pisia alle donne $\sigma$. non conviene

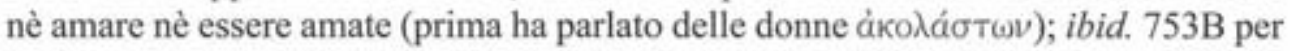

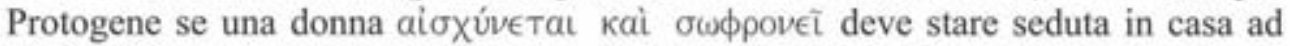
aspettare i suoi pretendenti; ibid. $753 \mathrm{C}$ ci si domanda se le $\sigma$. con il loro atteggiamento severo non abbiano nomea di insopportabili e odiose e se non le si chiami

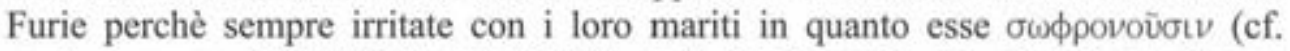
coniug. praec. 141F-142A); ibid. 759A ci si chiede quale dio agita l' entusiasmo

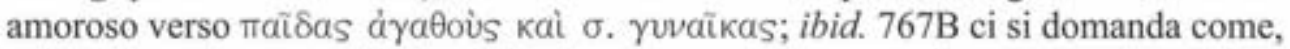
se il vizio può segnare il viso delle donne, non vi sia nessun lampo кобцíu каi $\sigma$.

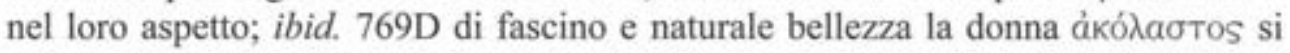
serve in vista del piacere e dell' inganno, la $\sigma$. per guadagnare l' affetto del marito; ibid. 769D come Platone consigliava al nobile e austero Xenocrate di sacrificare

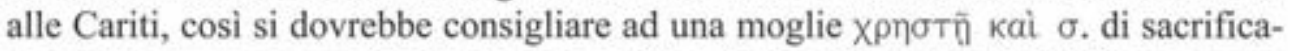
re ad Eros; an seni resp. 785D dopo la politica dedicarsi ad un' attività di poco conto è come togliere la veste ad una donna libera e $\sigma$. e darle un grembiule da ostessa; bruta anim. 988F Grillo dice ad Odisseo che egli ha fretta di sentire il suo discorso

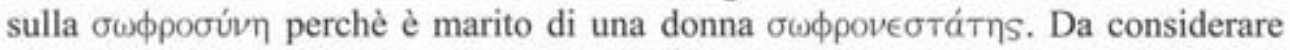
l'uso di $\sigma \omega \phi \rho o v i \zeta \epsilon t v$ in $L y c$. 14.2, dove, all' interno di un discorso sull' educazione delle fanciulle e sul matrimonio, si contesta l' affermazione di Aristotele che

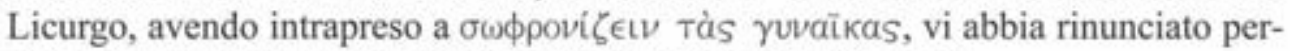

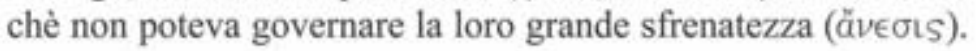

Non collegate con la sfera sessuale o dubbie si presentano sette attestazioni, riferibili a donne:

Thes. 26.4 un amico riferisce ad Antiope dell' amore di Soloente per lei; la donna

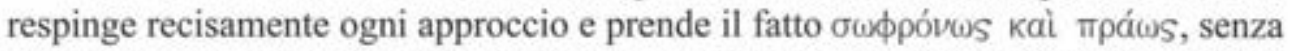
comunque accennarne a Teseo. Il contesto e la sottolineatura del rifiuto potrebbero 
autorizzare la tradizionale interpretazione, ma il collegamento con mpáws e l' accenno al silenzio con Teseo possono rimandare a un comportamento equilibrato; Pomp. 74.6 Cornelia, moglie di Pompeo, chiede perchè il marito non abbia abbandonato al suo triste destino colei che l' ha trascinato in una cosi grande sfortuna. Ella sarebbe stata felice se fosse morta prima di apprendere la morte del primo marito e

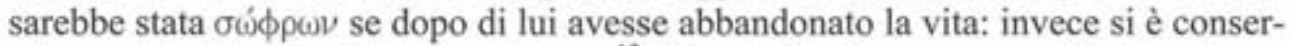
vata per fare il male di Pompeo Magno ${ }^{13}$. Si potrebbe leggere $\sigma$. nell' ottica di una moglie oltremodo fedele per cui la morte del marito è la fine di tutto, mentre lei si è unita in vincolo matrimoniale ad un altro uomo, a cui, come appare dalla Vita, fu legata da un profondo, reciproco amore; ma forse a $\sigma$. potrebbe essere connesso il significato di "sarei stata saggia, misurata, non avrei perseguito ambizioni"; T.G.C.G.1.6 alla morte del marito Cornelia si assunse il carico dei dodici figli e della

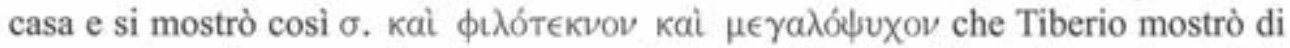
aver avuto ragione a morire al posto suo. Ella poi rifiutò l' offerta di matrimonio di Tolomeo e l' affiancamento al regno e allevò i figli Tiberio e Gaio cosi фL $\lambda \circ \tau_{i} \mu \omega \mathrm{S}$ che la loro educazione sembra aver avuto una parte più grande nella loro virtù che la loro natura. Qui l' insistenza sulla cura dei figli, della casa, sull' amore per i figli, sull' eccellente educazione ad essi impartita, rimandano per $\sigma \omega ́ \phi \rho o v a$ alla qualità di

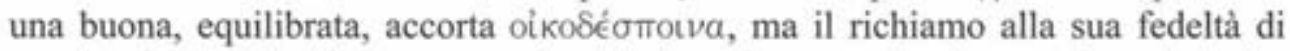
moglie, che rivolge il suo amore solo ai figli, può essere adombrato nella notizia che rifiutò l' offerta di un matrimonio importante ${ }^{14}$; in Ant. 2.1, dopo aver raccontato un episodio di Antonio padre, che, avendo dato un bacile d' argento a un amico bisognoso di soldi, al vedere la moglie arrabbiata e pronta a mettere sotto accusa i servi, confessò e chiese perdono, si spiega che la moglie era Giulia della casata dei Cesari,

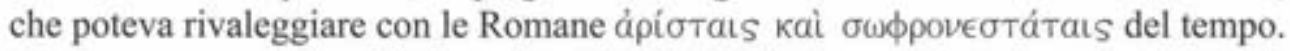
L' accenno successivo all' educazione dei figli da lei curata dopo la morte del marito e il successivo matrimonio, sembrano escludere il significato tradizionale a favore di quello più generico di "onesta", "temperante" in senso lato; in garr. 507B una

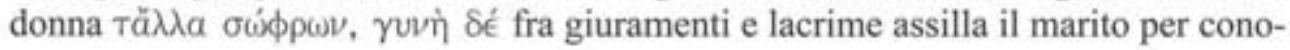

Per F. Le Corsu, Plutarque et les femmes, Paris, 1981, p. 42 non si vede perchè Cornelia fosse responsabile dei rovesci del suo sposo. Ma si puó ricordare che in Pomp. 55.4 si accenna a critiche relative al matrimonio fra Pompeo e Cornelia, legate sia alla forte differenza d' età fra i due, sia alla trascuratezza degli affari della città da parte di Pompeo in seguito alle nozze; in 55.7 si fa riferimento all' intromissione di Pompeo nella causa giudiziaria subita dal suocero Scipione Nasica, a cui in comp. Ages.-Pomp. 4.10 si attribuisce, secondo certe voci, di aver ingannato Pompeo, perchè, volendo appropriarsi delle somme riportate dall' Asia, le nascose, incitando Pompeo ad attaccare battaglia per una supposta fine dei fondi.

L' accenno al connesso rifiuto del regno potrebbe far pensare a una figura non ambiziosa. Per l' interpretazione tradizionale vd. comunque anche T.G-C.G. 25.6: Tiberio, rinfacciando a un nemico con fama di effeminato di avere insultato la madre Cornelia, gli disse che tutti i Romani sapevano che ella era stata lontano da un uomo piủ a lungo di lui, che era uomo. 
scere i segreti di una seduta blindata del senato, e il marito gioca sulla sua curiosità e tendenza al pettegolezzo ciarliero. Dumortier-Defradas traducono "honnête", ma più aderente al contesto forse Pettine che traduce "assennata": qui si fa riferimento alla "misura" che la donna in questo caso mette da parte per il piacere tutto femminile di spettegolare ${ }^{15}$; cons. ad $u x$. $609 \mathrm{~A}$ la donna $\sigma$. non soltanto deve resta-

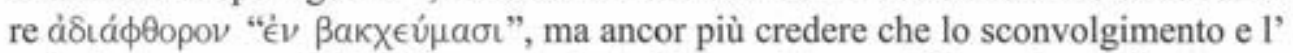

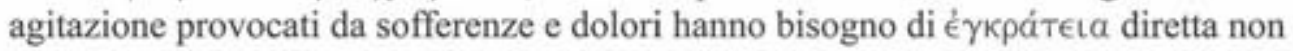

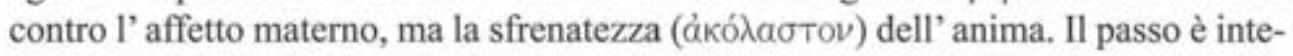
ressante perchè attribuisce a $\sigma$. un valore di sobrietà e di compostezza, doti che hanno caratterizzato finora il comportamento di Timossena, ma partendo da un

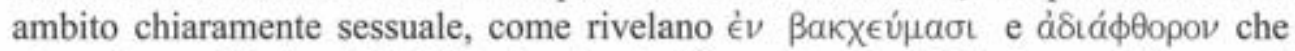

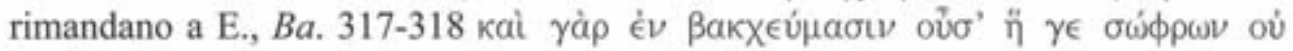

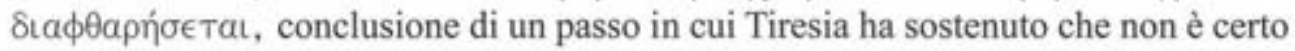

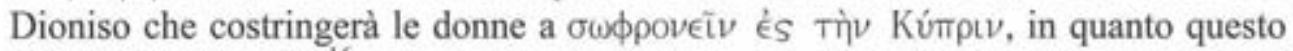
dipende dalla natura ${ }^{16}$. Senso di ordine e misura emergono anche da cons. ad $u x$. $609 \mathrm{E}$ in cui Plutarco ricorda a Timossena che pure in occasione della morte del primogenito ella aveva mostrato molta fermezza, tanto che dei forestieri, entrati in casa con Plutarco, avevano pensato che non fosse accaduta nessuna disgrazia così

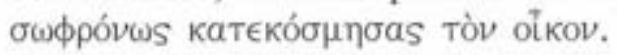

Considerando in senso più generale le attestazioni analizzate, possiamo notare in esse l' associazione di $\sigma \omega \dot{\phi} \phi \omega \nu, \sigma \omega \phi \rho \circ \sigma u ́ v \eta$ con alcuni aggettivi e sostantivi.

Ad esempio con quelli denotanti bellezza: Ant. 87.6 di Antonia lodata $\sigma \omega \phi \rho o-$

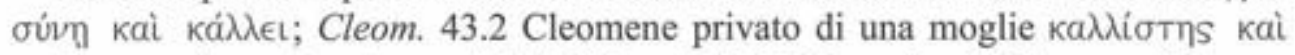

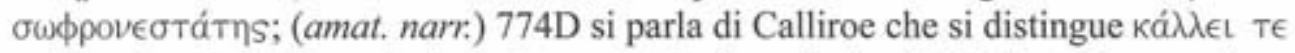

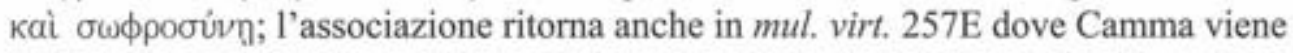
lodata non solo per la sua bellezza ma soprattutto per la sua virtù, tra cui la $\sigma \omega \phi \rho o-$ $\sigma u ́ v \eta^{17}$. Ci sembra che in questi casi la sottolineatura della bellezza valga nelle intenzioni dell' autore a mettere ancor più in risalto la virtù del personaggio, come può confermare coniug. praec. 141D, dove la padrona di casa, guardandosi allo specchio, è bene dica, se bella: "Che ne sarà di me, se saprò essere anche virtuo-

H. NoRTH, 1966, p. 206 n. 31 sostiene che Aristotele (Pol. 1277b.17-24) include il silenzio nella

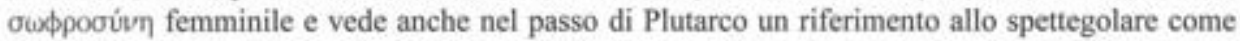
ákpacia e ákoda oía. Più chiaro il riferimento alla correttezza dei costumi per il silenzio in pubblico nel già considerato coniug. praec, 142C.

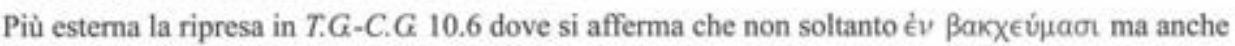

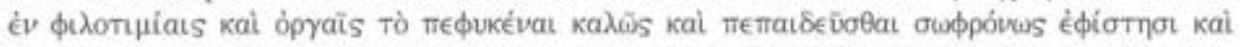

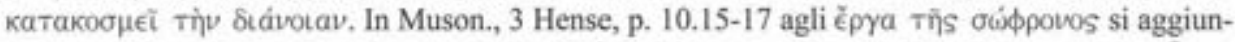

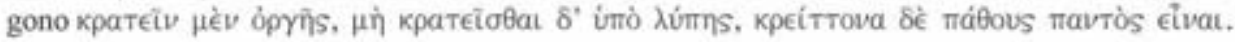
L' associazione bellezza e $\sigma$., e questo può essere interessante, torna anche per i fanciulli: vd. comp. Dem.-Ant. 4.5; (amat. narr.) 772E; con ulteriori doti Marc. 2.5; aet. Gr. 300D. 
sa?"; si può confrontare anche amat. 769D per la diversa utilizzazione della bellez-

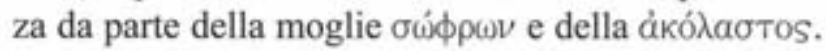

Con quelli denotanti nobiltà, in cui si ravvisa una spia della visione aristocratica di Plutarco, che sottolinea spesso i nobili natali: Sull. 35.9 Silla, se anche Valeria

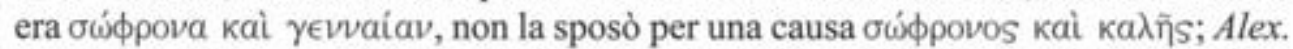

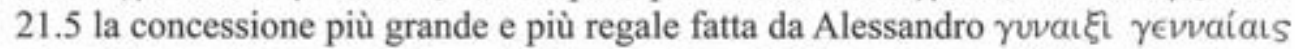

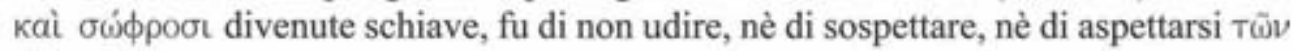
ai $\sigma \chi \rho \bar{\nu} \nu$.

Con quelli denotanti amore per il marito: mul. virt. 257E Camma, bella e ammi-

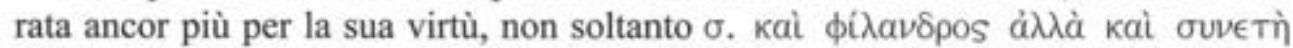
кaì $\mu \epsilon \gamma a \lambda o ́ \phi \rho \omega \nu$; è da notare che sono le prime due qualità che determinano la sua azione (avvelenamento di Sinorice, che, preso d' amore per lei, le aveva ucciso il marito): in amat. 768B-D la storia di Camma diviene rappresentativa del fatto che

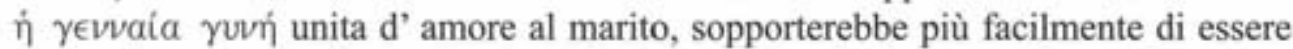
stretta da un orso o da un serpente piuttosto che lasciarsi toccare da un altro uomo

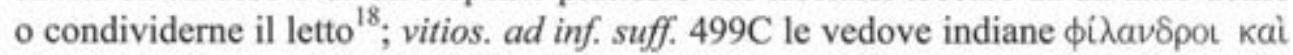

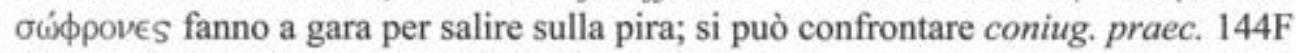
dove si dice che la moglie legittima a luce spenta non deve essere uguale a una qua-

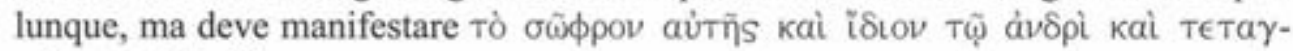

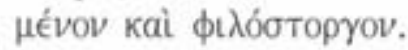

L' unione con l' aggettivo kóoutos aggiunge il decoro: coniug, praec, 140C il

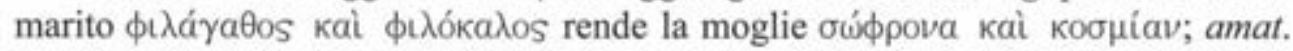
767B ci si domanda se, poichè il vizio può segnare il viso delle donne, nessun

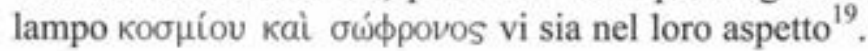

Che la $\sigma \omega \phi p o \sigma u ́ v \eta$, sessualmente parlando, sia rappresentativa delle donne come

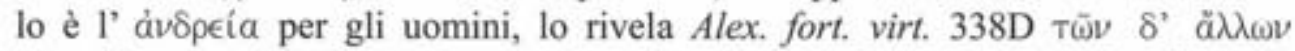

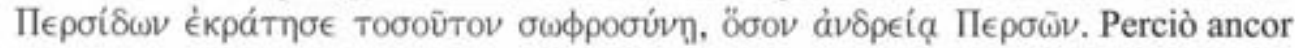
più risalta quando le due virtù si presentano unite in una donna. Così in Cat. Mi.

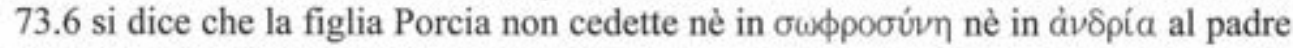
e si spiega che era sposata a Bruto alla cui congiura contro Cesare prese parte e che

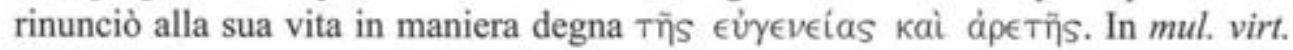

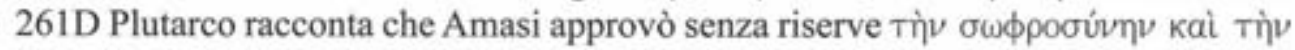

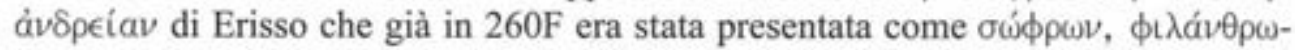
ToS e con parenti numerosi e potenti; essa finge di accettare la proposta di matrimonio del dissoluto Learco, uccisore del marito, e trasforma l' incontro amoroso in

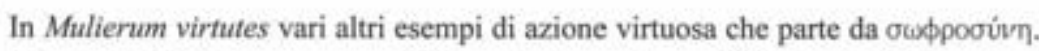

I due aggettivi ritornano associati in relazione ad uomini in Pyrrh. 4.7; adulat. 74B; (cons. ad Apoll.) 101F. 
un tranello omicida ${ }^{20}$.

La sfera di applicazione semantica di $\sigma \omega \phi \rho \omega \nu, \sigma \omega \phi \rho \circ \sigma u ́ \nu \eta$, si rivela più ampia per l' uomo. Vari sono gli esempi che rimandano alla temperanza sessuale ${ }^{21}$, ma anche alla temperanza in senso lato, relativa ai vari piaceri del corpo e di qui l' asso-

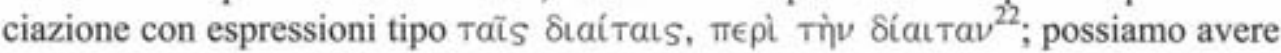
un' amplificazione della $\sigma$. in connessione con la semplicità di costumi, da cui l'

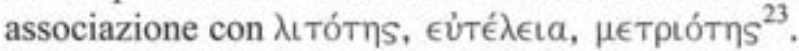

Ma abbiamo anche altre valenze.

Equilibrio: In Nic. 14.2 il fatto che Nicia si fosse opposto alla votazione della campagna in Sicilia senza lasciarsi esaltare dalle speranze, nè impressionare dalla

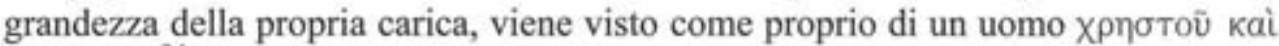
$\sigma \omega ́ \phi \rho \nu^{2} \mathrm{~S}^{24}$. In Dio 52.4 si dice che Dione volgeva la sua attenzione all'Accademia,

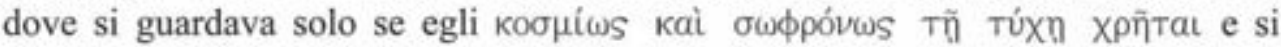

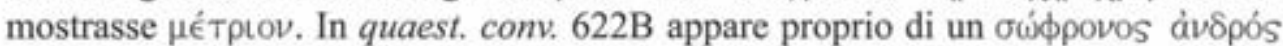
moderare le contese in un simposio. In ad princ. ind. 780D si sottolinea che diversa-

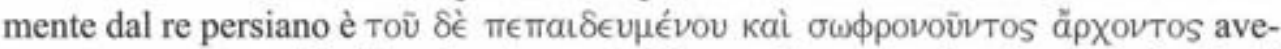
re entro se stesso chi gli parla e lo esorta. Da considerare anche Per: 11.1 , in cui gli aristocratici oppongono a Pericle, diventato potentissimo, Tucidide di Alopece, ăvઠ $\rho a$ $\sigma \omega \phi \rho \circ \nu \alpha$, che facendo opposizione a Pericle ridiede equilibrio alla vita politica ateniese.

Disciplina: In Brut. 36.2 si ricorda che Bruto dormiva poco per natura, ma aveva

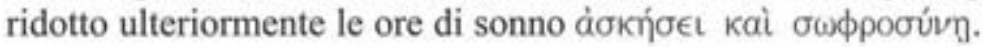

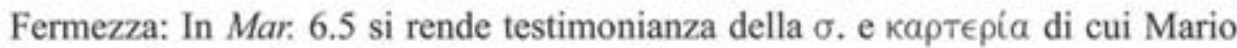
diede prova in occasione di un intervento chirurgico.

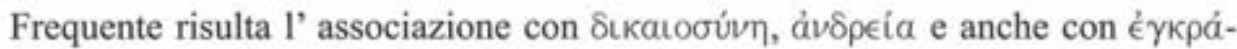
TELa.

Particolarmente interessante la descrizione del $\sigma$. in praec. ger. reip. $823 \mathrm{~A}-\mathrm{C}$ : egli non è un uomo rigido, ma cortese, affidabile, generoso, vicino ai dolori e e alle gioie

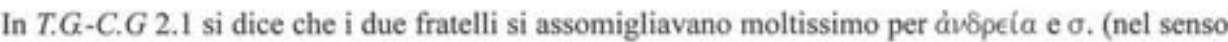
lato di vita modesta e moderazione nei piaceri, come si evince dall' intera vita), ma anche per generosità, eloquenza e grandezza d' animo.

21 Vd. ad es. comp. Arist.-Cat. Ma. 6.1; Ages. 14.1; Pomp. 53.2; Alex. 21.11; Arat. 51.4; coniug. praec. 139B.

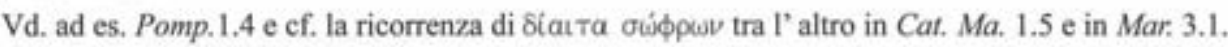

Vd. Cim.14.4; Crass. 1.2; Ages. 14.1; Cat. Mi. 3.10; comp. Agid.-Cleom. et T.G.C.G. 1.4; cf. Pomp. 18.3; con una connotazione solo di vita sobria e frugale: Dio 52.2 Dione generoso con gli

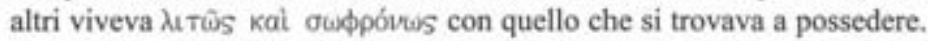


altrui, con un forte senso di misura nel rapporto con gli altri, "mostrando che egli vuole condurre la vita propria di tutti e essere un uomo come gli altri", "preoccupato del bene pubblico e pensante che la politica è un modo di vivere e di agire" 25 .

Se ritorniamo a considerare la $\sigma$. al femminile, potremo notare la frequenza delle ricorrenze nei Coniugalia Praecepta e nell' Amatorius, che potremmo far derivare dal tradizionale collegamento fra donna e sessualità e in vista della difesa del yévos; ma il valore che Plutarco attribuisce alla sessualità all' interno del matrimonio, non finalizzandola, come gli Stoici, alla procreazione di figli, ed inserendola invece nel gioco delle espressioni affettive fra i coniugi per la creazione di quell' unione armoniosa tra Eros ed Afrodite cosi fortemente esaltata nell' Amatorius $^{26}$, potrebbe anche rimandarci alla riabilitazione e nobilitazione che Plutarco fa della passione e all' utilità che alle passioni viene riconosciuta, in quanto le passioni, se misurate (e le virtù etiche sono proporzione e medietà di passioni ingenerate dalla ragione deliberativa e pratica), diventano naturali e insostituibili alleate della ragione, contribuendo a rafforzarla ${ }^{27}$.

Questo mi spinge a procedere ad un' analisi dei Coniugalia Praecepta volta ad individuare una linea interpretativa del matrimonio da parte di Plutarco, che si differenzi dal quadro di eccessiva novità attribuitagli da Foucault, dall' interpretazione tradizional-popolare della Patterson, con la sua forte insistenza su Omero, e dalla rienunciazione dell' interpretazione tradizionale di Nikolaidis.

Innanzitutto notiamo che nei Coniugalia Praecepta, seppure molto di tradizionale si mantiene nei rapporti della moglie con l' esterno e con quanto è estraneo al

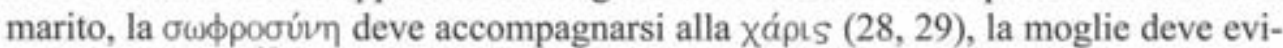

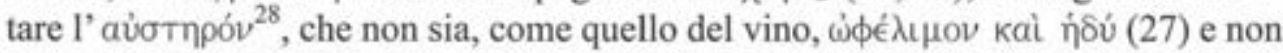

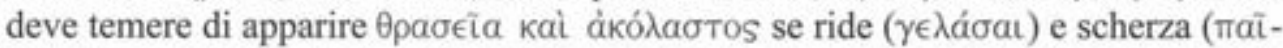
$\xi a i$ TL) col marito (29), ed i mariti a loro volta devono intrattenersi gioiosamente

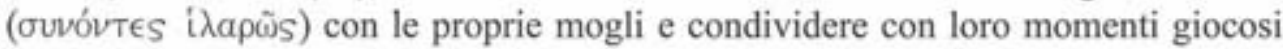

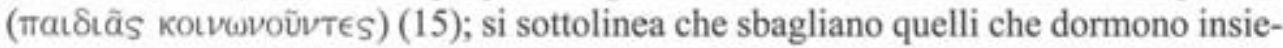
me in vista del piacere, ma dormono in letti separati quando sono in collera e in lite, non ricorrendo allora più che mai ad Afrodite (38), e si esortano i coniugi ad evitare gli attriti, e questo soprattutto a letto, ricordando che i dissapori, le offese e i

25

26

27

28

Cf, praec, ger. reip. $824 \mathrm{D}$ in cui il politico $\sigma$, appare come persona equilibrata, conciliatrice di discordic.

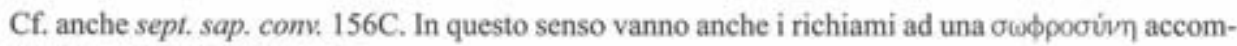
pagnata da Xápıs: vd. infra.

Vd. particolarmente Plu., virt. mor. capp. IV, V e XII. Sulla matrice peripatetica del tema dell' utilità della passione vd. F. BECCHI, 1990, p. 227. Da considerare inoltre l' importanza del piacere e

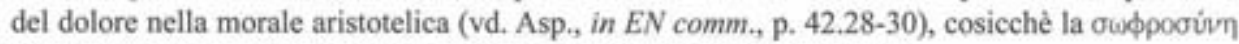
viene posta, quale regolatrice degli appetiti, alla base del sistema etico.

Cf. amat. 753C. 
risentimenti che genera il letto non è facile farli cessare in altro luogo e in altro momento (39); ed ancora, se la $\sigma \omega \dot{\phi} \rho \omega \nu$, quando si toglie la tunica, si veste del

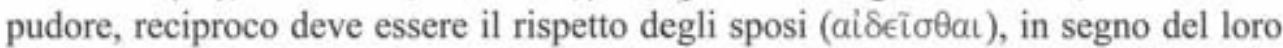

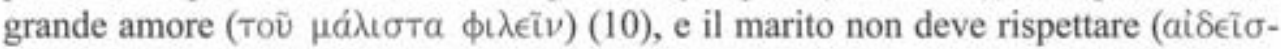

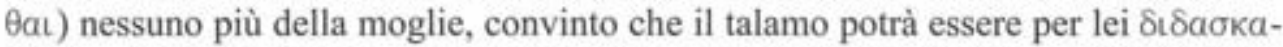

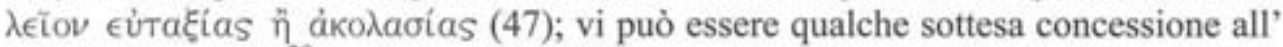

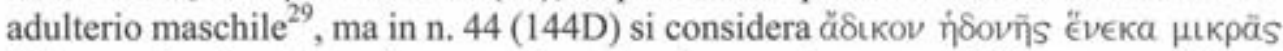

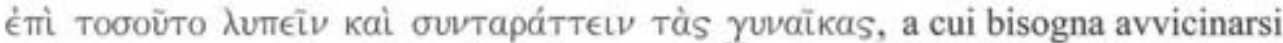
puri e non contaminati da relazioni con altre ${ }^{30}$; e in n. 42 (144B) si definisce ó

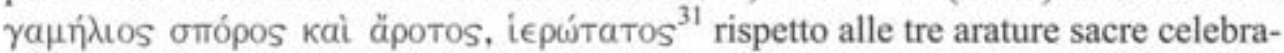
te dagli Ateniesi e mentre si invitano entrambi gli sposi a servirsene con rispetto

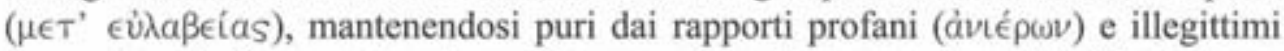

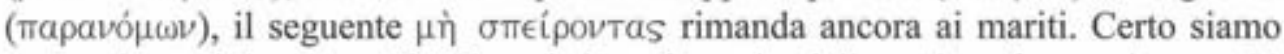

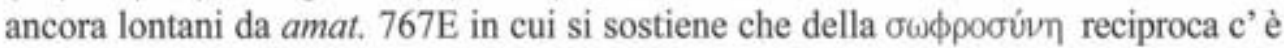
soprattutto bisogno nel matrimonio e quella che è legata a costrizioni o a leggi è impo-

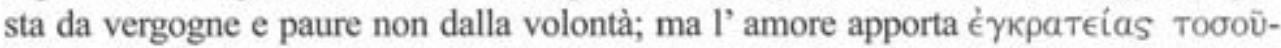

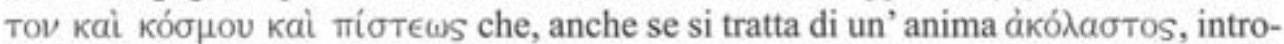

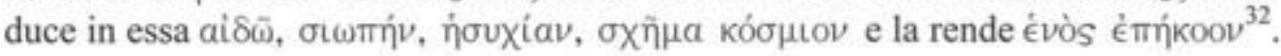

Consideriamo alcune immagini chiave e alcuni precetti attraverso cui si esprime

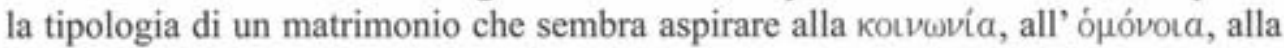
piena condivisione di beni, di sentimenti $(\sigma \cup \mu \pi a ́ \theta \in \iota a)$, affetti, amicizie come anche di credenze religiose: coniug. praec. 11 (139C) "come quando si odono due note suonare in accordo ( $\sigma u ́ \mu \phi \omega \nu$ ot), la melodia è data da quella più grave ( Toũ $\beta a \rho v-$

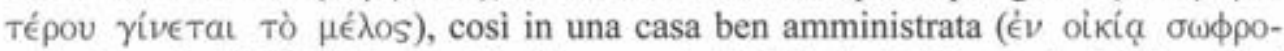

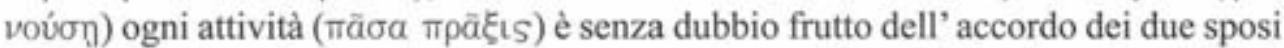

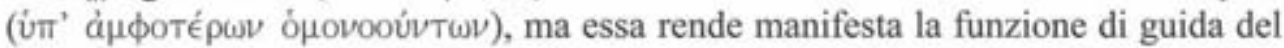

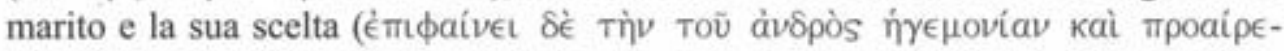

Cf. coniug. praec. 16 (140B), ma vd. infra.

Notare il giusto accento che A.G. NiKoLAIDIS, 1997, p. 73 n. 172 pone sul fatto che Plutarco veda qui I' infedelta "as infliction of a wound that makes the wife suffer". Si potrebbe confrontare il passo plutarcheo con Arist., Pol. 1335b.39, dove si pone come regola che il manifesto adulterio

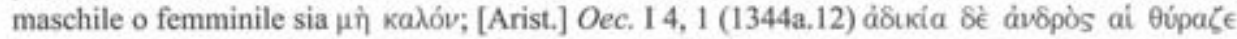

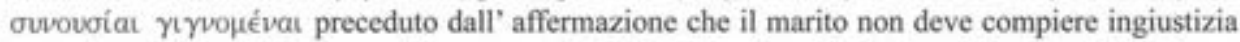
verso la moglie, cosi da non subire a sua volta ingiustizia; Oec. III 2 , dove si insiste sulla reciproca fedeltả, ma mentre in Plutarco è ingiusta la sofferenza fatta patire alla sposa, qui la fedeltà del marito viene vista come un onore dovuto sobriae mulieri. Vd. anche M. Foucautr, 1985, p. 175. Considerare l' uguale uso di $\lambda$ treiv in relazione ad un adulterio maritale in coniug. praec. 143F.

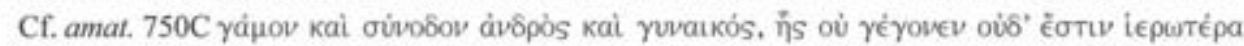

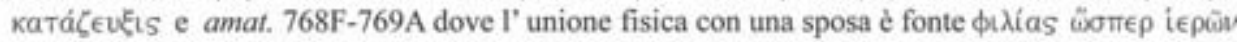

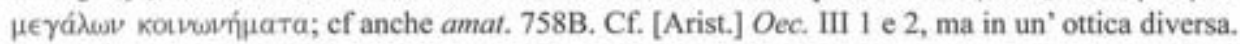


$\sigma เ v)$ "; ibid. 14 (140A) "come secondo i geometri le linee e le superfici non si muovono per se stesse, ma si muovono con i corpi, cosi bisogna che la moglie non abbia

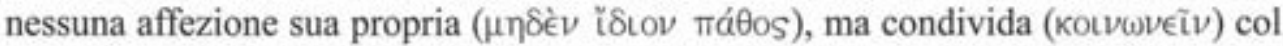
marito momenti seri e giocosi, di preoccupazioni e di risa"; ibid. 15 (140A) si dice che anche i mariti devono intrattenersi (бUvóvt€S) gioiosamente con le proprie

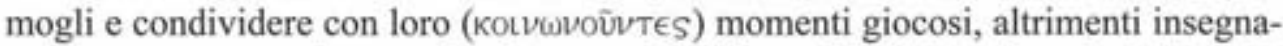

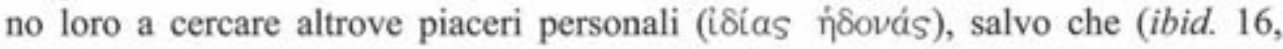
140B) un marito intemperante e sregolato deve tener lontano dalle sue eventuali intemperanze e sregolatezze la moglie, che non deve prendersela, in quanto è una forma di rispetto verso di lei ${ }^{33}$; ibid. 19 (140C-D) si ribadisce una kotvwvía che va dalla moglie verso il marito in quanto la moglie non deve avere isious $\phi i$ גous, ma condividere quelli del marito (Koเvoĩs $\delta \dot{\epsilon}$ X $\rho \tilde{\sigma} \sigma \theta a \mathrm{~L}$ ), e così pure deve onorare e riconoscere gli dei in cui crede il marito ${ }^{34}$; ibid. 20 (140E) "(come i medici sostengono che le ferite del lato sinistro si ripercuotono, per la diffusione del dolore, sul lato destro), cosi a maggior ragione è bene che la moglie provi le stesse affezioni del marito $(\sigma \nu \mu \pi a \theta \in i \nu)$ e il marito della moglie, affinchè come i nodi si rafforzano reciprocamente intrecciandosi, cosi, ricambiando ciascuno dei due l' affetto ( $T \eta \dot{v} v$

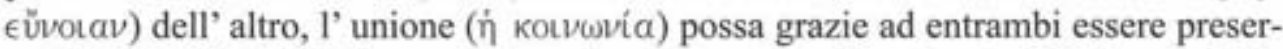
vata"; ibid. 20 (140F) la comunione è estesa anche ai beni materiali: "come chia-

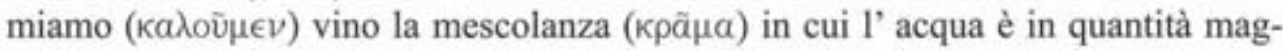
giore, così si deve dire $(\delta \in \tilde{\imath} \lambda \hat{\epsilon} \gamma \in \sigma \theta a \mathrm{~L})$ che il patrimonio e la proprietà immobiliare sono del marito anche se è maggiore il contributo della moglie" ${ }^{35}$; ibid. 34 (142EF), riprendendo una distinzione operata dai filosofi Stoici, e più esattamente da Crisippo $^{36}$, secondo cui vi sono vari tipi di unione, si afferma che questo si può

Un unico esempio: quello di Laide. Poi dopo generici richiami a serve e servi che, se in preda ad amore, rifiutano le profferte di padroni e regine, si introduce la storia di Camma con I' affermazione che una nobile donna unita d' amore al suo legale marito sopporterebbe più facilmente di essere stretta da un orso o da un serpente che essere toccata da un altro uomo o dividerne il letto.

Plutarco ricorda tra $\mathrm{l}^{\prime}$ altro in coniug. praec. 17 (140C) che il marito impronta ai suoi comportamenti buoni o cattivi quelli della moglie.

II rifiuto di rituali superstiziosi, di pratiche nascoste e furtive, di cui venivano tradizionalmente accusate le donne, viene spiegato con lo scarso gradimento da parte degli dei.

In amat. 752E Pisia, contrario al matrimonio della ricca Ismenodora col giovane Baccone, afferma che è una gran cosa se un giovane unendosi ad una fanciulla modesta €ís taùròv ì kpãois

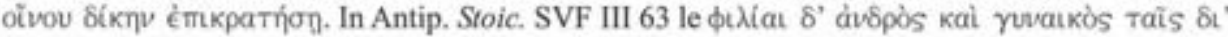

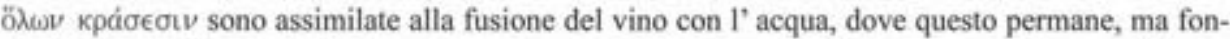

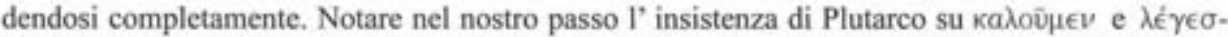
$\theta a$, che potrebbe rimandare alla polemica contro la posizione stoica relativa alla mescolanza totale: cf. Plu., comm. not. 1077F-1078B.

Vd. R. Klaerr, Plutanque, Oeuvres Morales, t. II, Paris, 1985, p. 317 n. 2. Cf. Plu., def. orac. 426 A.

${ }^{37}$ Per un significato più pregnante di oupфuris cf. Theophr., HP 5.2,4; Plu., Lyc. 25.5; in Plu., amat. 
applicare ai vari tipi di matrimonio per cui "quello di persone che si amano forma

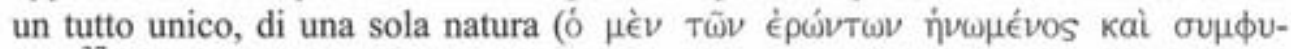
$\eta$ 's) ${ }^{\text {,37 }}$; ibid. 34 (142F-143A) "come, secondo i naturalisti, i liquidi si fondono in

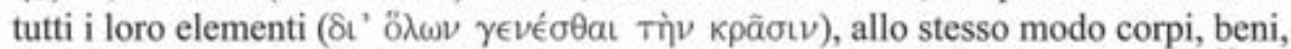
amicizie e relazioni dei coniugi devono amalgamarsi tra loro $(\dot{\alpha} \nu \alpha \mu \iota \chi \theta \tilde{\eta} \nu a t), 38$; in 48 (145D-E) si parte dall' affermazione che, seppur mai nessuna donna generò senza

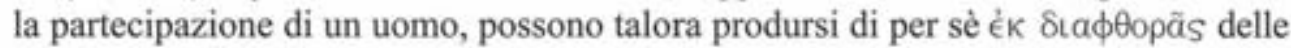
escrescenze carnose: questo bisogna evitare che si verifichi nell' anima delle donne, perchè se esse non ricevono semi di buone dottrine e non partecipano coi loro mariti al

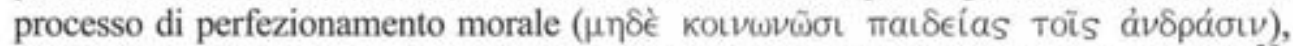

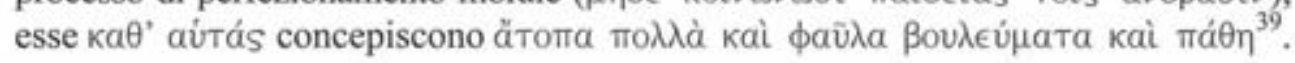

I testi analizzati complessivamente ci parlano di armonia e di unità, ma sembrerebbero mostrare anche un rapporto squilibrato a sfavore della moglie.

Abbiamo qui semplicemente il rimando alla tradizionale subordinazione della moglie al marito ${ }^{40}$ ? Perchè allora tanta insistenza sull' unità?

Guardiamo quanta importanza assuma nella trattazione il riferimento all' $\eta \eta_{0} \theta$ : coniug. praec. 4 (138F) "(l' amore impetuosamente originato nei giovani sposi dall' attrazione fisica e dall' età in fiore non bisogna considerare durevole e stabile,) a meno che, messe le sue radici nel comportamento e fatta presa sulla parte raziona-

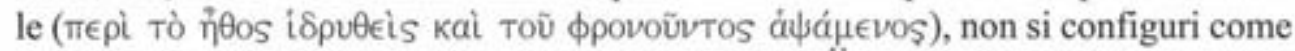

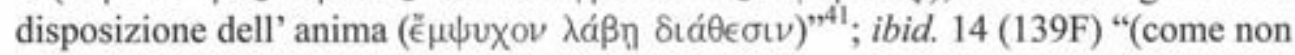
è di nessuna utilità uno specchio ornato che non riflette un' immagine fedele), così

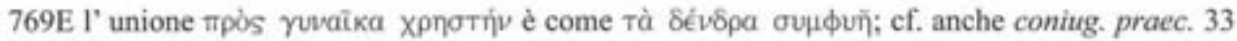

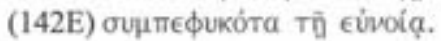

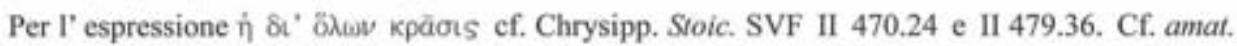

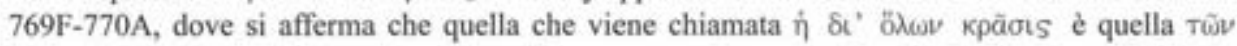

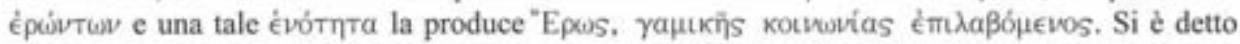
prima che l' amore all' inizio invece, come quando due liquidi si combinano ( $\sigma u \mu \pi \epsilon \sigma o ́ v T \omega v)$,

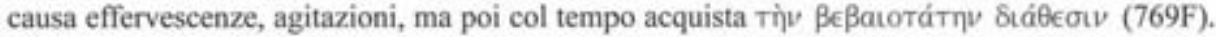

A queste parole segue l' esortazione ad Euridice di avere familiaritả con i precetti degli uomini saggi e dabbene e di avere sulla bocca le massime apprese da Plutarco, per dare gioia al marito ed essere

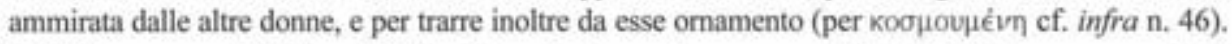

A.G. Nikol.Aidis, 1997, pp. 79-80 ritiene che in coniug. praec. 14 (140A) Plutarco non neghi la personalita della moglie, ma che la sua retorica, o meglio il suo amore per le similitudini (cf. la stessa similitudine in adulat. $63 \mathrm{C}$ ), lo abbia portato ad "a unintentional overstatement" e che, se egli può essere accusato di ritenere di regola gli uomini superiori alle donne, sono i requisiti e le qualità morali che danno agli uomini questa superiorità, non il loro genere, e lo scrittore non esita a raccomandare ai mariti la subordinazione a donne che li sopravanzino in tali qualità.

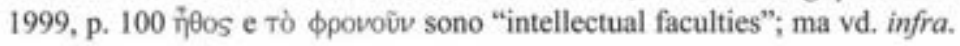


non reca nessun vantaggio una moglie ricca se non adegua la propria vita (Tòv ßíov

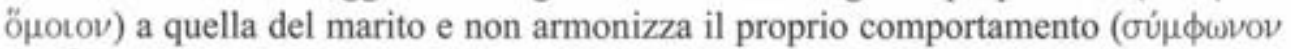
Tò $\left.\eta \theta_{0}\right)$ ) con quello di lui"; ibid. 22 (141A) "(Occorre che la moglie non faccia assegnamento su dote, nobiltà di nascita, bellezza,) ma riguardo a quelle cose in cui

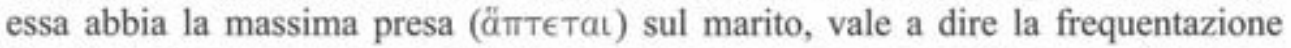

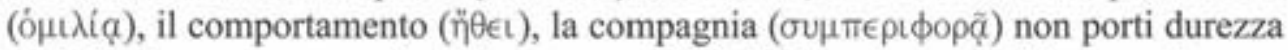
nè irritazione, ma armonia, gaiezza, amabilità (єủáp $\sigma \phi(\lambda \tilde{\eta}))^{\text {,42 }}$; ibid. 23 (141C) "(così una sposa legittima diventa una creatura irresistibile se, assommando tutto nella propria persona, dote, nascita, magici filtri e perfino la cintura di Afrodite,) riesce a conquistare col suo comportamento e la sua virtù

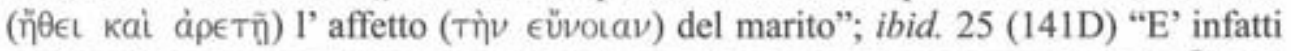

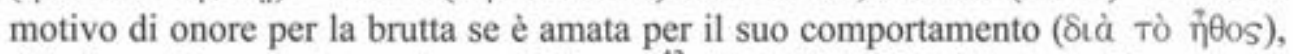
più che se fosse amata per la sua bellezza" ${ }^{43}$; ibid. 29 (142B) "Pertanto bisogna che anche la padrona di casa, poichè rifiuta ed evita giustamente tutto ciò che sa di eccessivo, di licenzioso e di ostentato, tanto più lavori sulle grazie del suo compor-

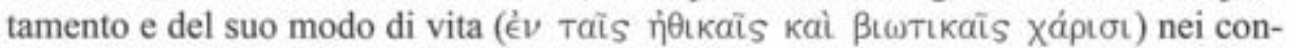
fronti del marito, creando così in lui un' abitudine alla virtù insieme col piacere ( $T \tilde{\varphi}$

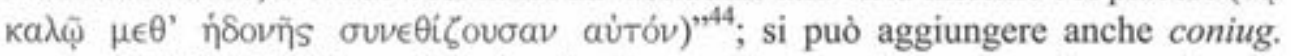

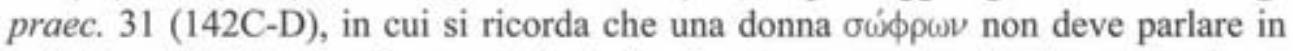
pubblico, nè di fronte ad estranei: infatti nella sua voce, se parla, si possono intra-

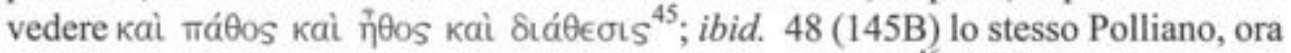

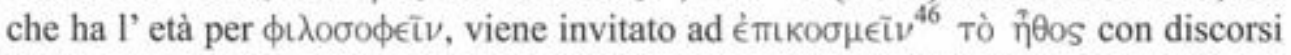
fondati su prove e ragionamenti, raccogliendo poi da ogni parte per la moglie Tò

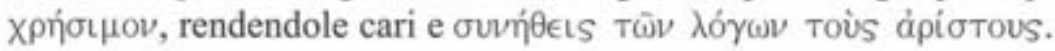

Consideriamo il passo introduttivo all" opera (138C): "Questi argomenti ... ora li invio a voi due quale dono comune, pregando le Muse di assistere e coadiuvare

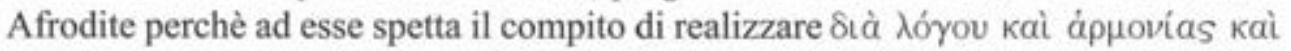

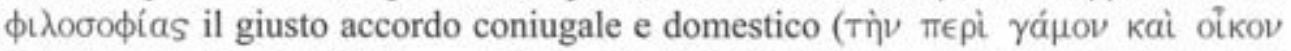

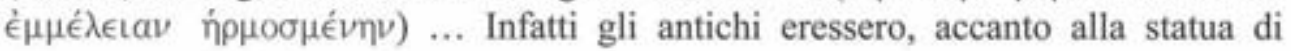

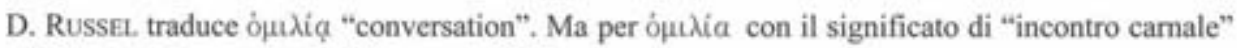
vd. Plu., coning, praec. 144B e amat. 756E.

Per la superiorità dell' ỉjos sulla bellezza vd. amat. $766 \mathrm{~F}$.

44 A.G. NiKol.Aids, 1997, p. 64 scrive in relazione al passo: "A woman leading a man toward good-

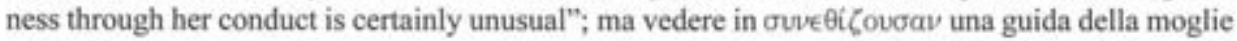
mi sembra eccessivo; qui si esprime la conseguenza del comportarsi con grazia di una oúфpus: 1" ingenerarsi di un' abitudine alla virtù, ed, elemento tralasciato da Nikolaidis, insieme col piacere; infine, per il marito che impronta ai suoi i comportamenti della moglie, basta considerare coniug. praec. $140 \mathrm{C}$ e $145 \mathrm{~A}$. 
Afrodite, quella di Ermes, nella convinzione che specialmente della ragione hanno bisogno i piaceri del matrimonio (

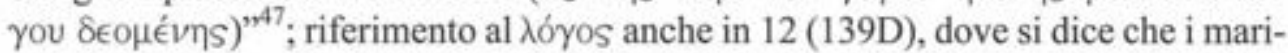
ti non riescono con le maniere forti ad allontanare le mogli da mollezza e lusso, "ma

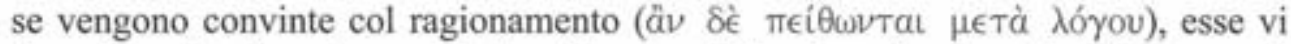

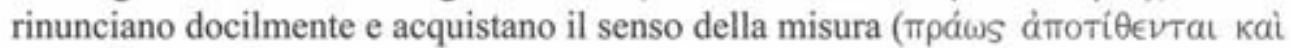
$\mu \in T \rho t a ́(o v \sigma \iota v) "$.

Teniamo presente l' insistenza su Toũ $\phi \rho \circ \nu$ oũ $\operatorname{Tos}^{48}$ in una connotazione tra morale e intellettuale in coniug. praec. $4(138 \mathrm{~F})$, già considerato in riferimento all'

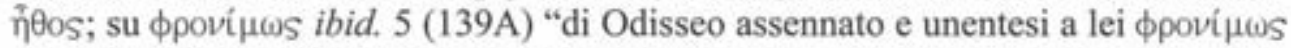
la maga si innamorò fortemente" e su фpóvıนos ibid. 6 (139A) "Le donne che preferiscono esercitare la loro autorità su uomini sciocchi, piuttosto che $\phi \rho \circ v i \mu \omega \nu$ ảkov́єเ $v$ "; riconsideriamo ibid. 11 (139C-D), in cui si afferma che in una casa ben amministrata ogni azione è il risultato dell' accordo dei due sposi, ma émı

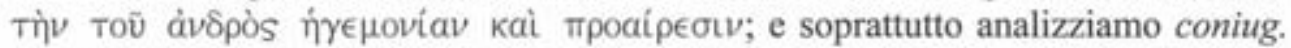

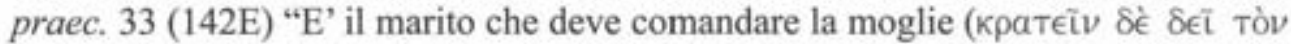

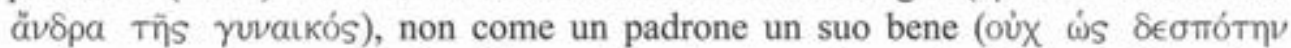

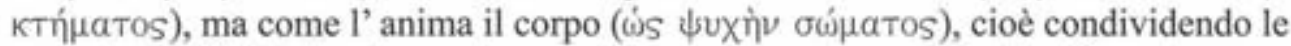
medesime affezioni e formando con lei una sola cosa grazie all' affetto ( $\sigma \nu \mu \pi \alpha-$

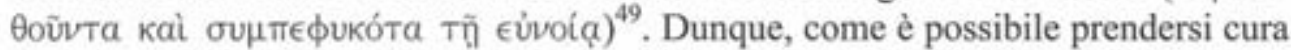

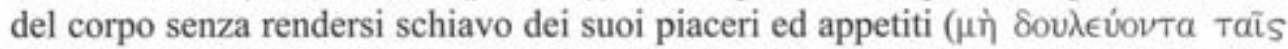

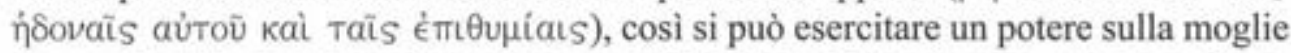

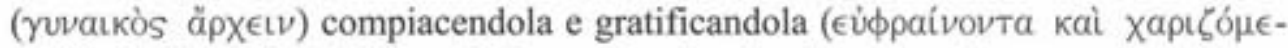

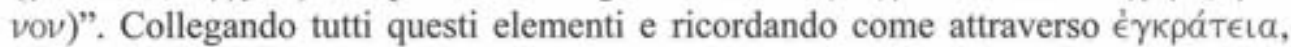

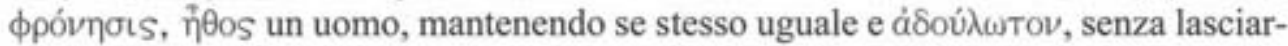

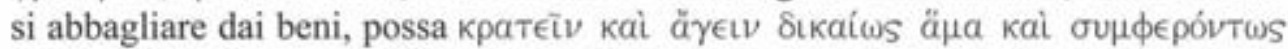
la donna bella o ricca che ha sposato (amat. 754B), mi sembra che all' interno di

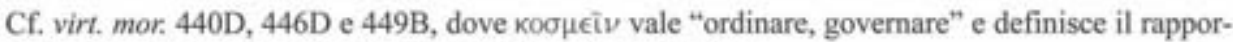

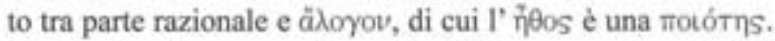

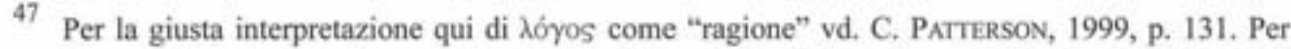
Hermes da interpretarsi come "ragione" vd. Is. et Os. 373B; cf. amat. 757B e fr. 200, 63-64 Sandbach. L. GoEsster, 1999, p. 98 scrive: " $\lambda$ óyos, by wich he means consideration and philosophical reflection". La generale interpretazione qui di $\lambda$ óyos è "parola, discorso".

Per tò фрovoūv nell' accezione di parte razionale dell' anima vd. superst. 166C; virt. mor. 445D, 447D, 451B; ser. mum vind. 563E, 564C, 566A; soll. anim. 961A; suav, viv. Epic. 1105A; fr. 200,

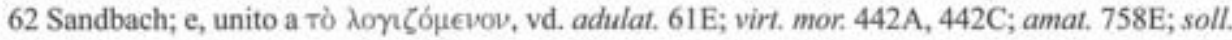

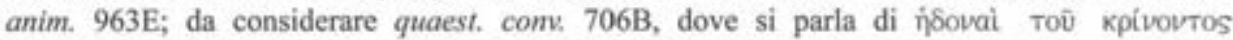
ámтópleval кaì фроиоûvтos.

49

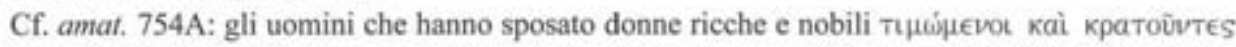

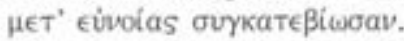


questo matrimonio filosoficamente impostato, come chiarito dall' introduzione e come sottolineato da un' adeguata terminologia, il rapporto moglie-marito o meglio

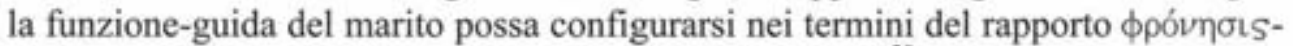
virtù etica quale soprattutto emerge dal De virtute morali ${ }^{50}$.

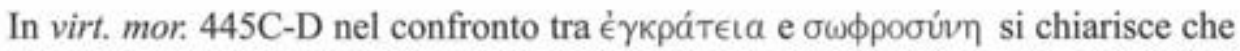

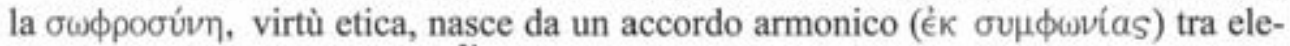
mento razionale e passionale ${ }^{51}$, accordo che sorge da una sottomissione spontanea

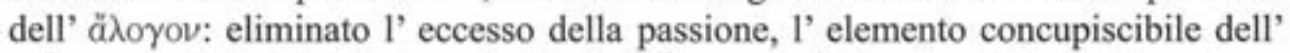

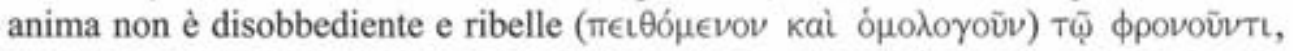
non arreca e non subisce molestie; già in 445B era stata delineata la consonanza tra ragione e passione che caratterizza l' animo del temperante attraverso l' immagine

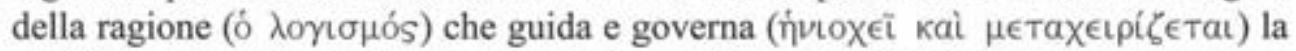

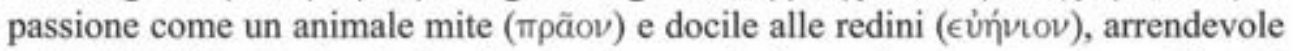

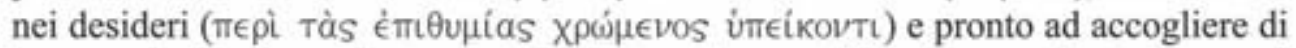
buon grado la misura giusta e conveniente; un' esemplificazione applicata ancora al temperante ritorna in $446 \mathrm{D}-\mathrm{F}$, dove si afferma che nell' animo del temperante regnano un perfetto equilibrio, una calma e una buona salute, che permettono all' elemento irrazionale, fornito di una straordinaria capacità di ubbidire docilmente

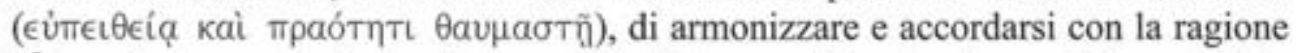

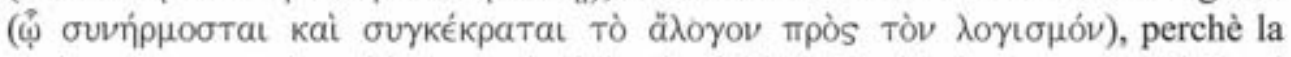
ragione ha spento i moti impetuosi, violenti e folli dei desideri, mentre quelli di cui la natura ha veramente bisogno li ha resi simpatetici (o $\mu$ oт $a \theta \tilde{\eta})$, obbedienti (úmíkoa) e pronti a collaborare amichevolmente nelle decisioni pratiche ( $\phi \dot{\lambda} \lambda a$ кaì

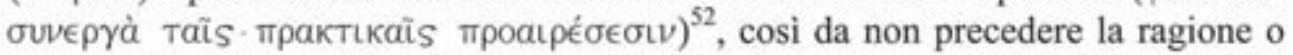
restare al di sotto o essere disordinati e disobbedienti, ma docili in ogni loro impul-

50

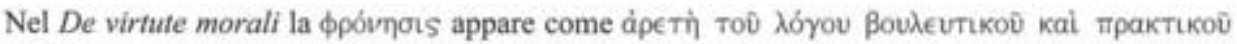

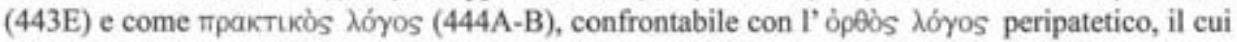
compito naturale è eliminare gli eccessi e le dissonanze delle passioni. Per la definizione di $\phi \rho o ́ v \eta \eta-$

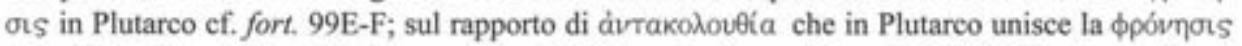
alle virtù etiche cf. fort. $97 \mathrm{E}$; in aud. poet. $32 \mathrm{C}$ in relazione a Hom. Od. 265-266, che ascrive I' iniziale retto comportamento di Clitemnestra ai suoi onesti pensieri, si afferma che il poeta $\tau \underline{\emptyset}$

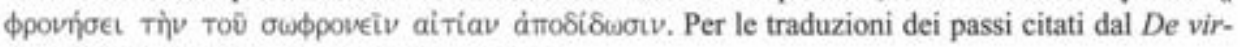
tute morali sono debitrice a F. BECCHI, 1990.

51

Cf. Asp., in EN comm., p. 35.23-24 per l'ó $\mu$ oфwovia di ragione e passione che caratterizza l' animo

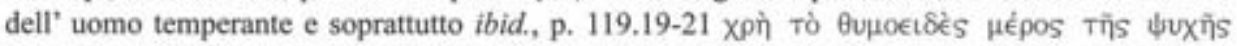

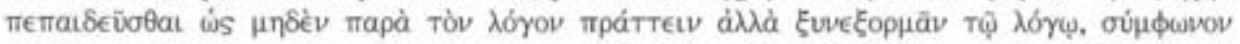

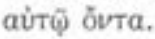

52

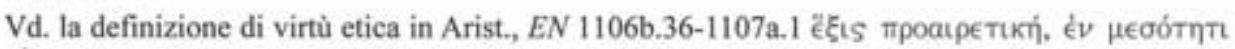

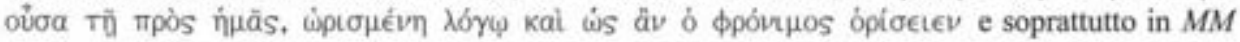

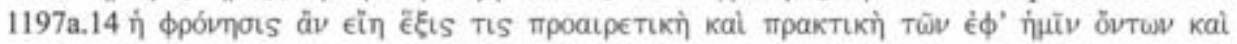

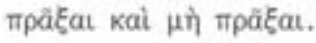


so, confermando il detto di Senocrate a proposito dei veri filosofi che essi soli fanno volentieri quello che tutti gli altri fanno controvoglia, costretti dalla legge. D'

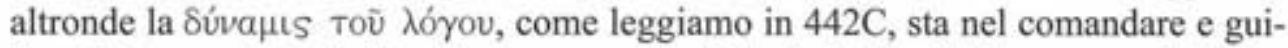
dare ( smare, di cedere e persuadere in modo più efficace di qualsiasi costrizione e forza. C' è d'altronde un' armonica conformazione dell' irrazionale alla ragione (Tò oup-

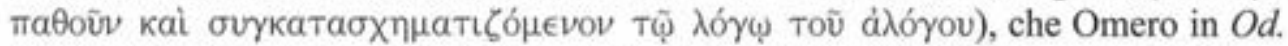
19.208-212 è riuscito ottimamente a rappresentare con Odisseo, che di fronte a Penelope piangente, sottomesso al giudizio, tratteneva il respiro e il sangue e le

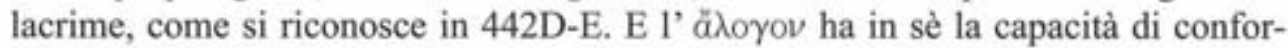

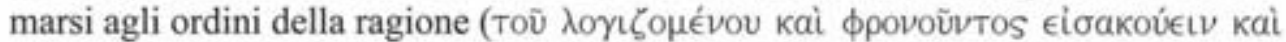

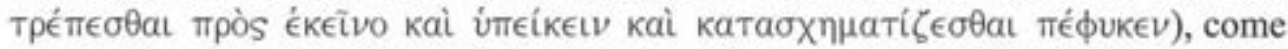
emerge da 442B-C. E per natura è la sua obbedienza alla ragione: così in 443B-C si legge che la facoltà irascibile e appetitiva dell' anima, sede dell' afflizione e della

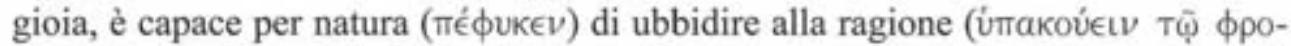

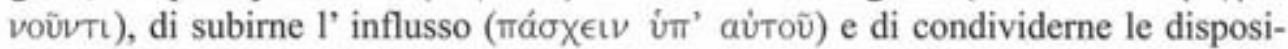

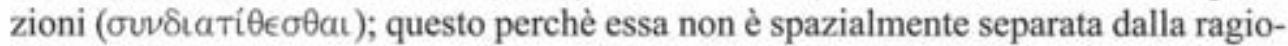
ne, nè plasmata dall' esterno e non ne subisce l' impronta sotto la spinta della necessità o della forza, ma per natura è sempre unita ad essa, sviluppandosi insieme in un

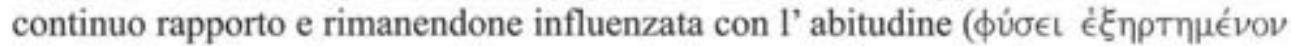

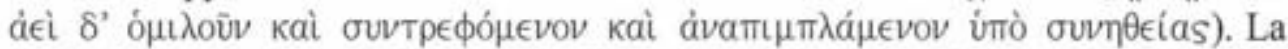
stessa idea ritorna in $450 \mathrm{E}$ in cui si afferma che la natura vuole che la ragione, che

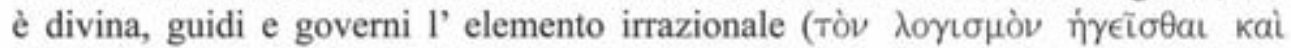

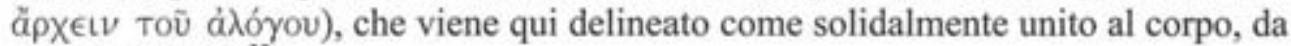
cui trae origine ${ }^{53}$.

E' inoltre da sottolineare la funzione svolta dall' ñoos nello svilupparsi della virtù etica.

Nel sopra citato virt. mor: $443 \mathrm{C}$ c' era il riferimento al fatto che la facoltà irascibile e appetitiva dell' anima rimane influenzata dalla ragione (Tò фpovoũ $\nu$ ) con

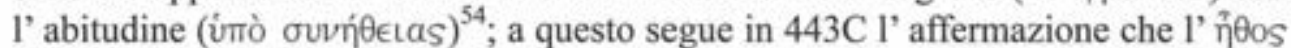

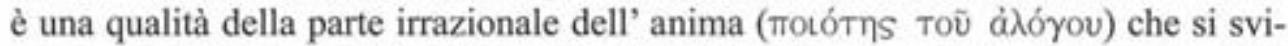
luppa con l' abitudine (

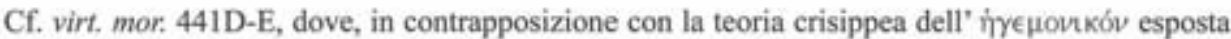
in $441 \mathrm{C}$, si afferma che neppure Pitagora ignorava come sia proprio dell' anima avere al suo interno qualcosa di composto e formato di due nature diverse, poichè l' irrazionale come un secondo

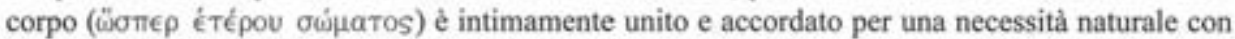

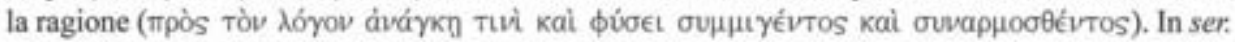

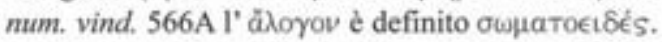

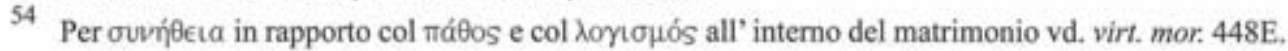




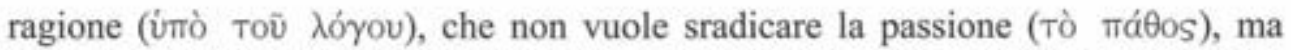

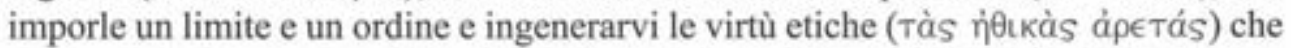
sono proporzione e medietà di passioni; e la ragione mediante la saggezza ( $\tau \bar{n}$

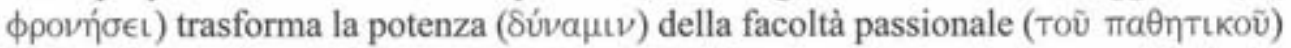

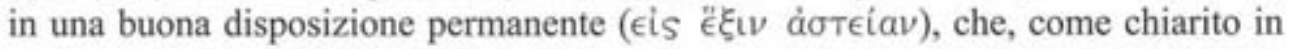
virt. mor. 443D, diventa vizio o virtù secondo che la passione sia stata educata (

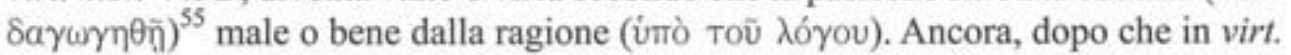
mor. 444A sono stati definiti i due momenti essenziali dell' azione etica della $\phi \rho o ́ v \eta-$

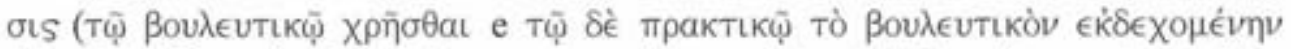

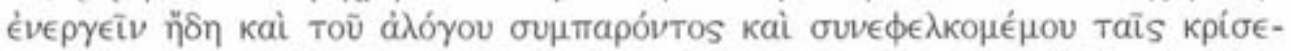
$\sigma \iota v)$, si sottolinea in virt. mor: 444B che i giudizi hanno bisogno dell' ópuń ed è la

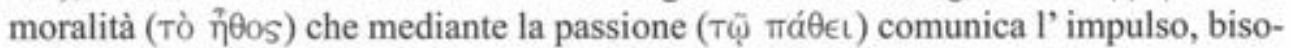
gnoso che la ragione gli imponga un limite (

Se il raffronto è valido si capisce come il matrimonio per eccellenza sia quello

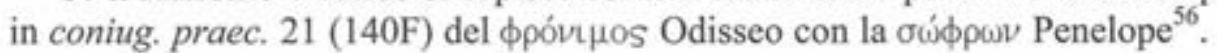

Nell' opera plutarchea, oltre al passo considerato e al citato amat. 754B, manca-

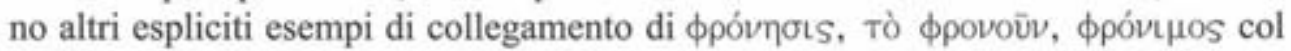
matrimonio; abbiamo invece un chiaro esempio di supremazia femminile all' interno del matrimonio, e quindi non possiamo più parlare di supremazia di genere: questo si verifica grazie al $\phi \rho \circ v \in \tilde{\imath} v$, in una connotazione non esplicitamente intellettuale, per Ismenodora nell' Amatorius.

Plutarco risponde alle obiezioni sollevate da Pisia (752E-F) sul matrimonio di un

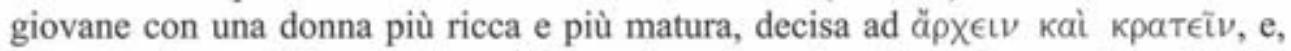
venendo al caso di Ismenodora afferma, dapprima, che stimare in una donna la ricchezza più che la virtù o la nascita è basso e sconveniente, ma fuggire la ricchezza unita alla virtù e alla nascita è da sciocchi $(754 \mathrm{~A})$, poi, che Ismenodora più di una fanciulla riuscirà a consacrare le sue cure al giovane Baccone, perchè i giovani sono

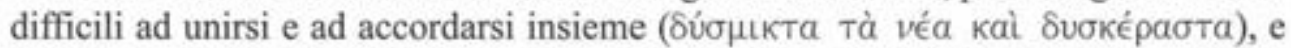

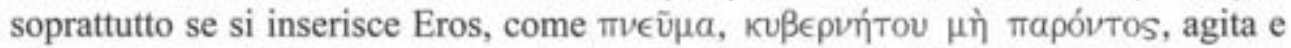

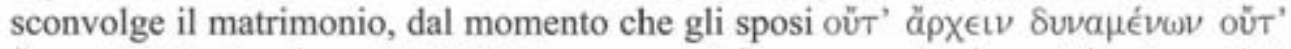

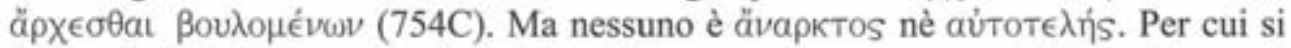

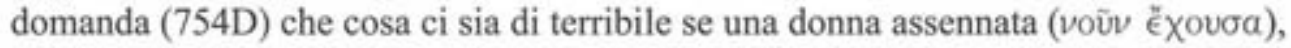

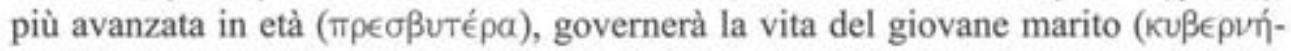

Per la passione bisognosa di educazione vd. anche virt. mor. 451C.

L. GoESSLER, 1999, p. 104 in riferimento al passo scrive "Here the two qualities exemplified by Odysseus and Penelope correspond with each other precisely: for prudence (sôphrosynê) is the female version of good sense (phronêsis) ", ma i confronti con la tradizione epica e soprattutto con Plu., brut. anim. 988F e 989A mi sembra autorizzino la mia interpretazione. Vd. anche R. KLAERr, 1985 , p. 153 "Ulysse pratiquait la prudence, Pénélope la pudeur". 


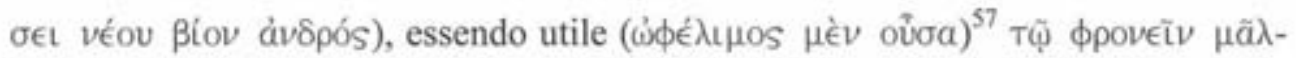

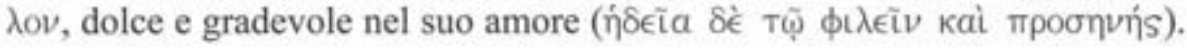

Insieme al фpovєĩ $\nu$, che si associa a dolcezza e gradevolezza di affetti, è l' età, come è evidente dal contesto, che giustifica la sua supremazia; d' altronde il colle-

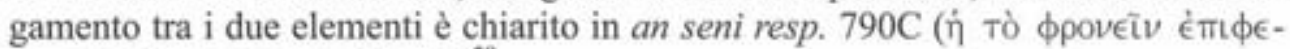

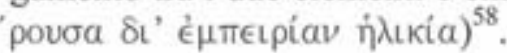

Ad altre donne, non molte, è attribuito il $\phi \rho o v \in \bar{\nu} \nu$ come qualità (intellettuale?) tra altre, ma questo non sembra decidere per una supremazia femminile.

In mul. virt. 255E Aretafila viene descritta come figlia e moglie di $\gamma \nu \omega \rho i ́ \mu \omega \nu$

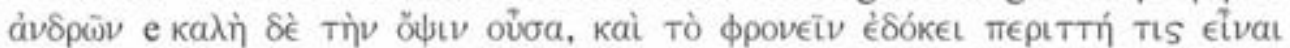

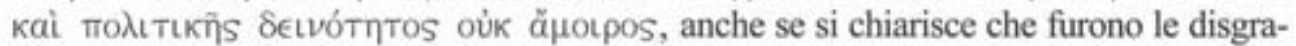
zie della sua patria a renderla illustre. Agi infatti per liberare la sua patria dalla tirannide, anche se attraverso sotterfugi, inganni, sfruttando le altrui debolezze, dando però anche prova di coraggio e forza d' animo, salvo poi ritirarsi nel gineceo, dedita ad attività femminili; ma se il tiranno Nicocrate, che l' aveva costretta al matrimonio, dopo averle ucci-

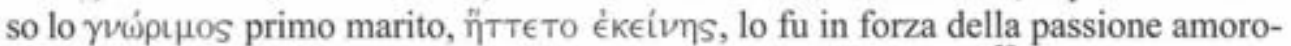
sa che lo legava a lei, come sottolineato varie volte da Plutarco ${ }^{59}$.

Misteriosa resta per noi la vita di Abrote, moglie di Niso, che Plutarco presenta

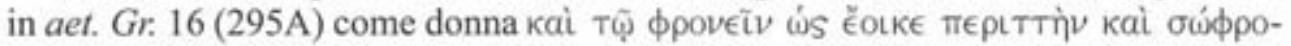
va $\delta\llcorner a \phi \epsilon \rho o v ́ T \omega s$, da cui, dopo la sua morte, prese il nome per volontà del marito una veste che ella era solita portare; niente tuttavia si chiarisce della tipologia del suo rapporto matrimoniale.

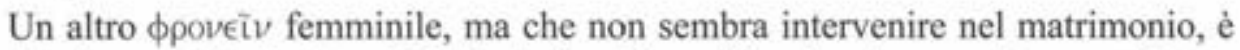
quello riconosciuto in Rom. 1.2 alla troiana Rome (

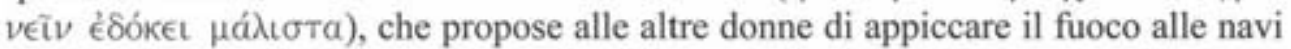
per costringere gli uomini a fermarsi stabilmente alla foce del Tevere; quelli, trovatisi li bene, resero onore a Rome, e quindi alla sua decisione, e da lei, ús aitías, diedero il nome alla citta ${ }^{60}$.

Per un comando che deve unire in sè l' utilità cf. amat. 754B; anche in coniug. praec. 48 (145B) Polliano, che ha ormai I' età per $\phi \lambda \lambda_{0} \circ \phi \in i v$, deve raccogliere da ogni parte per la moglie tò

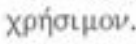

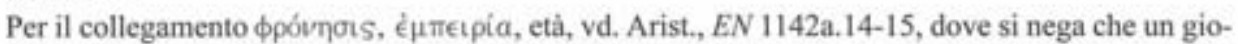

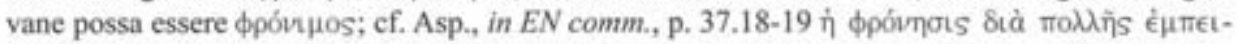

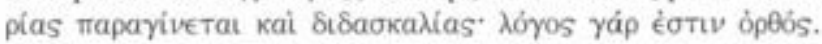

59 Vd. 255F, 256A, 256B, 256D.

60

In relazione alla stessa vicenda, in $m u l$. virt. 1 (243E-244A) riflessione e decisione sono attribuite a tutte le donne troiane: Rome è ricordata solo come la prima donna ad aver appiccato il fuoco. La vicenda viene ripresa in aet. Rom. 6 (265B-E), dove I' incendio delle navi viene visto come un atto d' audacia delle donne troiane. 
Di Cleopatra si dice genericamente (Ant. 25.5) che ella incontrò Antonio in un'

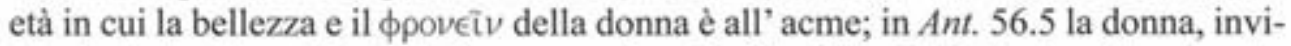
tata da Antonio a ritirarsi dalla guerra, incarica Canidio di dirgli che oltre al resto

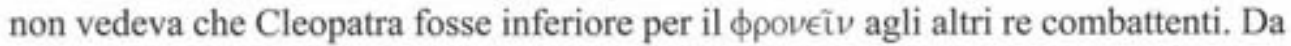
considerare che alla presentazione della moglie di Antonio, Fulvia, piena di volontà di dominare, segue in $10.6 \mathrm{l}$ ' affermazione che più tardi Cleopatra fu debitrice a Fulvia delle lezioni di sottomissione alle donne che questa aveva impartito ad Antonio; ma se nel rapporto fra Cleopatra e Antonio questi risulta spesso suo succube, come lo stesso Plutarco non manca di sottolineare, si ravvisa da parte di Antonio una dipendenza legata ai piaceri ${ }^{61}$.

Concludendo, si può comunque riconoscere come in questi ultimi casi, con l'

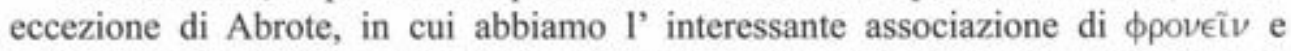
$\sigma \omega \phi \rho \circ \sigma u ́ v \eta$, il riferimento al $\phi \rho \circ \nu \in \bar{\tau} \nu$ precede il momento in cui un' azione femminile, che coinvolge direttamente o indirettamente l' altro sesso, ottiene un risultato positivo.

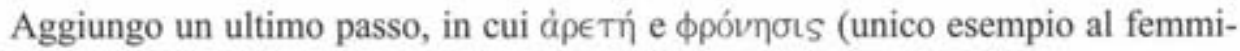
nile) meritano la deferenza maschile: Pyrrh. 4.6 in cui si dice che Pirro, ancora $\mu \in \mathrm{L}-$

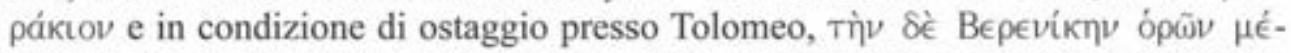

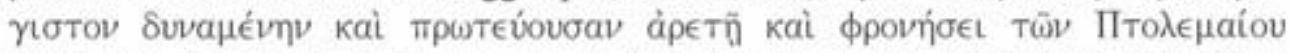

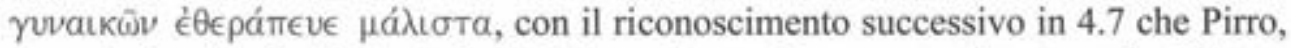

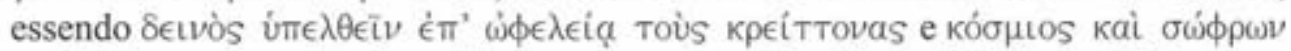

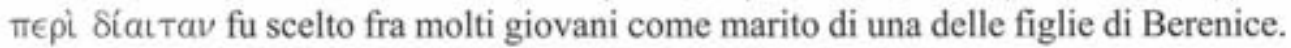

\section{BiBLIOGRAFIA}

BABBrT, F.C.,

- Plutarch's Moralia, vol. III, Cambridge (Mass.), 1931.

BECCHI, F,

- Plutarco. La virtù etica, Napoli, 1990.

BOULOGNE, J.,

- Plutarque. Oeuvres Morales, t. IV, Paris, 1981.

FOXHALL, L.,

- "Foreign Powers. Plutarch and Discours of Dominatio in Roman Greece", in S.B. Pomeroy (ed.), Plutarch's Advice to the Bride and Groom and A Consolation to His Wife, New York-Oxford, 1999, pp. 138-150.

61

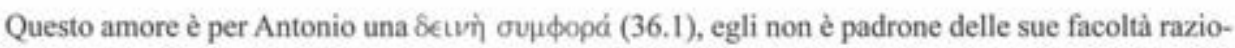
nali, come fosse sotto I' influenza di filtri e sortilegi (37.6), anche se in 27.3 si dice che la bellez-

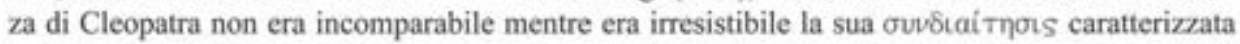
dal fascino della conversazione, modo di trattare con gli altri, piacevolezza della voce, capacità di parlare con molti nella loro lingua. 
Foucault, M.,

- La cura di sè. Storia della sessualità 3, tr. it., Milano, 1985, pp. 179-209.

GOESSLER, L,,

- "Advice to the Bride and Groom: Plutarch gives a Detailed Account of His Views on Marriage", in S.B. Pomeroy (ed.), Plutarch's Advice to the Bride and Groom and A Consolation to His Wife, New York-Oxford, 1999, pp. 97-115; (vd. GoEssLer, L., Plutarchs Gedanken über die Ehe, Zürich, 1962, pp. 44-69).

HENSE, O.,

- C. Musonii Rufi Reliquiae, Lipsiae, 1905.

Nikolaidis, A. G,

- "Plutarch on Women and Marriage", WS, 110 (1997), 27-88.

NORTH, H.,

- Sophrosyne: Self-Knowledge and Self-Restraint in Greek Literature, Ithaca-New York, 1966.

PATterson, C.,

- "Plutarch's Advice to the Bride and Groom, Traditional Wisdom through a Philosophic Lens", in S.B. Pomeroy (ed.), Plutarch's Advice to the Bride and Groom and A Consolation to His Wife, New York-Oxford, 1999, pp. 128-137; (vd. PATterson, C., "Plutarch's Advice on Marriage: Traditional Wisdom through a Philosophic Lens", in ANRW 33.6, Berlin-New York, 1975, pp. 4709-4723).

RUSSEL, D.,

- "Advice on Marriage", in Plutarch: Selected Essays and Dialogues, Oxford, 1993, pp. 284-296.

Stadter, Ph.A.,

- Plutarch's Historical Methods: An Analysis of the Mulierum Virtutes, Cambridge (Mass.), 1965.

- "Philosophos kai Philandros. Plutarch's View of Women in the Moralia and the Lives", in S.B. Pomeroy (ed.), Plutarch's Advice to the Bride and Groom and A Consolation to His Wife, New York-Oxford, 1999, pp. 173-182. 


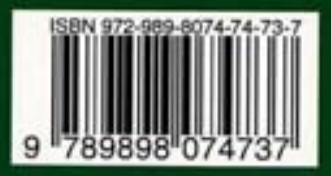

